\title{
Reduced TUBA1A Tubulin Causes Defects in Trafficking and Impaired Adult Motor Behavior
}

\author{
DeGeorgia Buscaglia, ${ }^{1}$ CKyle R. Northington, ${ }^{1}$ Jeffrey K. Moore, ${ }^{2}$ and Emily Anne Bates ${ }^{1}$
}

https://doi.org/10.1523/ENEURO.0045-20.2020

${ }^{1}$ Department of Pediatrics, University of Colorado Anschutz Medical Campus, Aurora CO 80045 and ${ }^{2}$ Department of Cell and Developmental Biology, University of Colorado Anschutz Medical Campus, Aurora, CO 80045

\begin{abstract}
Newly born neurons express high levels of TUBA1A $\alpha$-tubulin to assemble microtubules for neurite extension and to provide tracks for intracellular transport. In the adult brain, Tuba1a expression decreases dramatically. A mouse that harbors a loss-of-function mutation in the gene encoding TUBA1A $\left(T u b a 1 a^{N D /+}\right)$ allows us to ask whether TUBA1A is important for the function of mature neurons. $\alpha$-Tubulin levels are about half of wild type in juvenile Tuba1a $\mathrm{ND/}^{\mathrm{N}}$ brains, but are close to normal in older animals. In postnatal day $(\mathrm{P}) 0$ cultured neurons, reduced TUBA1A allows for assembly of less microtubules in axons resulting in more pausing during organelle trafficking. While Tuba1 $\mathrm{a}^{\mathrm{ND/+}}$ mouse behavior is indistinguishable from wild-type siblings at weaning, Tuba1a $\mathrm{ND/+}^{\mathrm{mice}}$ develop adult-onset ataxia. Neurons important for motor function in Tuba1a ${ }^{\mathrm{ND/+}}$ remain indistinguishable from wild-type with respect to morphology and number and display no evidence of axon degeneration. Tuba1a ${ }^{N D /+}$ neuromuscular junction (NMJ) synapses are the same size as wild-type before the onset of ataxia, but are reduced in size in older animals. Together, these data indicate that the TUBA1A-rich microtubule tracks that are assembled during development are essential for mature neuron function and maintenance of synapses over time.
\end{abstract}

Key words: ataxia; microtubule network; movement disorder; trafficking; TUBA1A; tubulin

\section{Significance Statement}

Defects in axonal trafficking have been reported in models of movement disorders, but it has been unclear whether trafficking defects are coincident or causative. Our results suggest that deficits in TUBA1A $\alpha$-tubulin can cause trafficking defects that impair synaptic maintenance leading to a movement disorder without axon degeneration or impacting myelination or neuron survival.

\section{Introduction}

Microtubules are the tracks on which proteins, RNA, and organelles are trafficked. Microtubule polymers are composed of $\alpha \beta$-tubulin heterodimers. In humans, nine $\alpha$-tubulin and $10 \beta$-tubulin isotypes are differentially expressed and could influence properties of specific cellular microtubule networks (Gadadhar et al., 2017). In developing neurons, the most highly expressed $\alpha$-tubulin is encoded by Tuba1a (Lewis et al., 1985; Miller et al., 1987; Gloster et al., 1994, 1999; Zhang et al., 2014). Heterozygous mutations that disrupt human TUBA1A are associated with severe

\footnotetext{
Received February 10, 2020; accepted March 3, 2020; First published March 12, 2020.

The authors declare no competing financial interests.
}

brain malformations, termed tubulinopathies, suggesting that TUBA1A is critical for brain development (Keays et al., 2007; Kumar et al., 2010; Cushion et al., 2013; Aiken et al., 2017). The most common human TUBA1A variants dominantly disrupt microtubule function in neurons (Tian et al., 2010; Aiken et al., 2019b). No deletions, frameshift, or nonsense mutations have been identified in association with TUBA1A tubulinopathy patients (Fallet-Bianco et al., 2008; Cushion et al., 2013; Oegema et al., 2015). We recently proposed TUBA1A mutations that impair brain development dominantly disrupt microtubule function, while

Author contributions: G.B., J.K.M., and E.A.B. designed research; G.B., K.R.N., performed research; G.B., K.R.N., analyzed data; J.K.M., and E.A.B. edited the final manuscript; G.B. wrote the paper. 
heterozygous TUBA1A loss-of-function mutations are tolerable for brain development and are not clinically reported (Aiken et al., 2019a). How TUBA1A contributes to adult neuron function has not been reported.

TUBA1A constitutes $\sim 95 \%$ of neuronal $\alpha$-tubulin mRNA developmentally (Miller et al., 1987). While Tuba1a expression is reduced overall in the adult brain, some regions including the hippocampus, cerebellum, basal ganglia, and certain regions of the cerebral cortex retain TUBA1A expression in adulthood (Bamji and Miller, 1996). Over time, neuronal microtubule networks acquire post-translational modifications (PTMs) and associations with microtubuleassociated proteins (MAPs) that stabilize neuronal microtubules (Brady et al., 1984; Yan et al., 1985; Audebert et al., 1994; Bonnet et al., 2001; Song et al., 2013; Natarajan et al., 2017). TUBA1A proteins may incorporate into microtubules during neurodevelopment and remain within the microtubule network of adult neurons. Indeed, TUBA1A, and other tubulins, have a half-life on the order of weeks to months in rodents (Heo et al., 2018). TUBA1A may be important in the adult neuronal microtubule network. However, mechanistic understanding of TUBA1A function in vivo has been limited by the high degree of sequence similarity between $\alpha$-tubulin isotypes, as isotype-specific $\alpha$-tubulin antibodies are not available.

To overcome the technical limitations to studying the specific impact of TUBA1A in adult neurons, we use the Tuba1a $^{N D}$ mutant mouse which harbors an asparagine to aspartic acid substitution at amino acid residue 102 (Gartz Hanson et al., 2016). Homozygous Tuba1a ${ }^{N D / N D}$ mice are neonatal lethal and have severe brain malformations consistent with homozygous Tuba1a ${ }^{\text {null }}$ and Tuba1a quas mutant mice (Bittermann et al., 2019) and similar to phenotypes observed in human patients with heterozygous TUBA1A mutations (Gartz Hanson et al., 2016; Aiken et al., 2017). The corresponding ND substitution in the primary $\alpha$-tubulin in yeast decreased $\alpha$-tubulin protein levels, altered microtubule dynamics, reduced incorporation of the mutant $\alpha$-tubulin into the lattice, and impaired mitotic division (Gartz Hanson et al., 2016). Together, these data demonstrate that Tuba $1 a^{N D}$ substitution results in less stable microtubules. In contrast to the severe impact of Tuba1a $a^{N D / N D}$ homozygous substitution on neuronal development, heterozygous Tuba1 $\mathrm{a}^{\mathrm{ND} /+}$ mice survive to adulthood (Gartz Hanson et al., 2016). The Tuba1 $a^{N D /+}$ heterozygous mice can be used to interrogate the requirement for TUBA1A neuronal microtubules within the mature brain.

This work was supported by the University of Colorado Movement Disorder Center (E.A.B.), the Department of Cell and Developmental Biology (J.K.M.), and the National Institutes of Health Grant 5R01GM112893-05 (to J.K.M.).

Acknowledgements: We thank Dr. John Caldwell for his assistance dissecting muscle fibers and supplying the rhodamine-labeled bungarotoxin, Danae Mitchell for helping with the spinal cord sectioning and staining, and Dr. Jayne Aiken for insightful conversations.

Correspondence should be addressed to Emily Anne Bates at emily.bates@ cuanschutz.edu.

https://doi.org/10.1523/ENEURO.0045-20.2020

Copyright $\odot 2020$ Buscaglia et al.

This is an open-access article distributed under the terms of the Creative Commons Attribution 4.0 International license, which permits unrestricted use, distribution and reproduction in any medium provided that the original work is properly attributed.
Here, we show that reduced function of TUBA1A, a tubulin isotype that has historically been characterized only in a developmental context, impacts the ability to maintain synapses over time and ultimately results in an adultonset movement disorder. Microtubule tracks are not adequately assembled when there is a deficit in TUBA1A tubulin early in development. Insufficient microtubule tracks result in organelle trafficking deficits that are apparent in Tuba1 $\mathrm{N}^{\mathrm{ND} /+}$ heterozygous neurons early in development. Despite microtubule track abnormalities and resulting changes in trafficking, Tuba1 $a^{N D /+}$ microtubules are sufficient to support survival and morphologically normal neuromuscular junction (NMJ) synapses. However, NMJ synapse morphology and animal behavior deteriorate in an age-related manner in Tuba1 $a^{N D /+}$ animals, without evidence of neuronal cell death or degeneration. Together, these data indicate that both developing and adult neurons require functional TUBA1A, and suggest that the "developmental" tubulin TUBA1A is required for mature neuronal function.

\section{Materials and Methods}

\section{Mice}

All animal research was performed in accordance with the Institutional Animal Care and Use Committee at the University of Colorado School of Medicine. The Tuba $1 a^{N D}$ mouse model was generated via an ENU-induced mutagenesis forward genetic screen and is described in more detail in Gartz Hanson et al. (2016). All mice used were maintained on a 129S1/C57BI6 genetic background. Mice were kept on a 12/12 h light/dark cycle with ad libitum access to food and water. Tuba $1 a^{N D}$ and wild-type littermate mice were maintained on water supplemented with $0.2 \mathrm{~g} / \mathrm{l} \mathrm{MgSO}{ }_{4}$ to promote Tuba $1 \mathrm{a}^{\mathrm{ND} /+}$ survival and ability to reproduce. Male and female mice were represented in all studies. All mice were genotyped by PCR amplification of tail DNA followed by Sanger sequencing to differentiate homozygous or heterozygous Tuba1 $a^{N D /+}$ mice from wild type.

\section{Behavioral phenotyping}

Mice were assessed for abnormal motor function between two and 10 months of age. Mice were allowed to acclimate to the testing room for $30 \mathrm{~min}$ before behavioral assessment. Gait analysis testing was performed in an enclosed raised chamber with a strip of paper lining the bottom. Fore and hind paws were painted differing colors just before testing, after which mice were placed at the chamber entrance and allowed to run through. Distances between footprints taken from a running gait were analyzed in ImageJ (ImageJ; National Institutes of Health). Rotarod performance was assessed using a Rota-Rod Treadmill (Med Associates, Inc) apparatus with one mouse per lane and a maximum of four mice tested simultaneously. Mice received no prior training on the rotarod task. The rod initially began rotating at 3 RPM and increased until reaching a terminal rotational speed of 30 RPM after $3 \mathrm{~min}$. The time until each mouse fell off or lost its grip such that it could not remain upright on the rod 
was measured. A cutoff time of 4 min was established for any mice that remained on the rod after peak rotational speed was reached. Mice performed three subsequent trials on the rotarod per testing session, with a 15-min rest period in between trials. Grip strength was tested in mouse forelimbs using a grip strength meter (Stoelting Co). Three subsequent forelimb grip strengths were recorded per testing session.

Mouse hind-paw sensory function was tested between three and six months.

For both Von Frey and Hargreaves analyses, individual mice were contained in $8 \times 8 \times 17 \mathrm{~cm}$ chambers and were allowed to acclimate to the testing chamber for $30 \mathrm{~min}$. Mice were only tested when resting on all four paws in a non-grooming awake state. Von Frey analysis of mechanical stimulus sensation was performed in left and right hind-paws using Touch Test Sensory Probes (Stoelting Co). Von Frey performance score was analyzed using the SUDO method established in Bonin et al. (2014). Hargreaves analysis of thermal pain sensation was assessed using a paw thermal stimulator instrument (University of California, San Diego). Time until withdrawal from thermal stimuli was recorded for each animal in both left and right hind-paws. Three subsequent trials were performed for each hind-paw per testing session.

\section{Histology}

Mice were anesthetized and transcardially perfused with $0.1 \mathrm{M} \mathrm{NaCl}$ and $4 \%$ paraformaldehyde (PFA) for histology. Tissues of interest were dissected and postfixed in $4 \%$ PFA. Serial tissue sectioning was performed on a CM1520 cryostat (Leica) and 30- $\mu \mathrm{m}$ serial cryosections were obtained for all histology experiments. For all histologic analyses, three regions were chosen for quantification which spanned the anterior-posterior axis, and the same regions were assessed for each animal. For Nissl staining, sections from the lumbar spinal cord were stained using $0.1 \%$ cresyl violet. For immunofluorescence studies, PFA-fixed tissues were blocked in PBS containing 5\% bovine serum albumin (BSA) with $0.3 \%$ Triton X-100. Primary and secondary antibodies were diluted in PBS containing 1\% BSA with $0.1 \%$ Triton X-100. For $\alpha$-bungarotoxin labeling of NMJ synapses, PFA-fixed extensor digitorum longus (EDL), flexor digitorum brevis, and soleus muscles were dissected from the hindlimbs of postnatal day (P) 30 and adult mice. Muscle fibers were teased apart using forceps and mounted onto positively charged glass microscope slides. Muscle fibers were blocked in a blocking buffer containing 3\% BSA, 5\% goat serum, and $0.5 \%$ Triton $\mathrm{X}-100$ and then stained with primary antibodies diluted in blocking buffer at $4^{\circ} \mathrm{C}$ overnight. Secondary antibodies and rhodamine-conjugated $\alpha$-bungarotoxin (a generous gift from Dr. John Caldwell) were added for two hours at room temperature. Primary antibodies were as follows: mouse anti-calbindin D-28k (Swant AgCB10abs; 1:250), rabbit anti-ER81 (BioLegend 840401; 1:5000), mouse anti-SMI32 (BioLegend 801702; 1:5000), rabbit anti-synaptophysin (Invitrogen MA5-14532; $1: 200)$. Fluorescently conjugated secondary antibodies were Life Technologies all used at 1:500. For NMJ analysis, $Z$-stack images were obtained at $63 \times$ magnification with a slice size of $0.5 \mu \mathrm{m}$ with 10 total slices covering $4.5-\mu \mathrm{m}$ total z-distance.

\section{Electron microscopy}

Mice used for electron microscopy were perfused with $0.1 \mathrm{M} \mathrm{NaCl}$ and $2.5 \%$ glutaraldehyde $4 \%$ PFA, after which the spinal column was dissected and postfixed in $2.5 \%$ glutaraldehyde $4 \%$ PFA overnight at $4{ }^{\circ} \mathrm{C}$. Following postfixation, spinal cords were dissected from the spinal column, and a 2-mm region of the cervical spinal cord between $\mathrm{C} 2$ and $\mathrm{C} 4$ was dissected and sent for further processing and imaging by the CU School of Medicine Electron Microscopy Core facility. Myelin thickness, axon density, and axon diameter were measured from electron micrographs in the lumbar spinal cord. G-ratio was calculated using the formula $G=r_{i} / R_{i}$ where $r_{i}=$ radius of axon and $R_{i}=$ radius of axon and myelin. At least three sections per animal were analyzed, measuring 100 axons per animal. The same total area was quantified between wildtype and Tuba $1 a^{N D /+}$ mice.

\section{Cell culture and transfection}

Dissociated neurons were cultured from post-natal day 0 (P0) to P2 mouse cortices. Brains were removed and placed into HBSS (Life Technologies) supplemented with 1 M HEPES (Life Technologies) and $1 \mathrm{~mm}$ kynurenic acid (Tocris Bioscience). Meninges were removed and cortices were dissected and cut into $\sim 1-\mathrm{mm}$ pieces. Cortical pieces were triturated to a single-cell suspension using glass Pasteur pipettes. Cortical neurons were plated onto 35-mm Poly-D-Lysine coated glass-bottom culture dishes at a density of 350,000 cells $/ 35 \mathrm{~mm}$. Neurons were maintained in a $37^{\circ} \mathrm{C}$ humidified incubator with $5 \% \mathrm{CO}_{2}$ in phenol-free Neurobasal-A medium (Life Technologies) supplemented with B-27 (Thermo), penn/strep (Thermo), GlutaMax (Thermo), $5 \mathrm{ng} / \mathrm{ml} \beta$-FGF (Invitrogen), and sodium pyruvate (Thermo). For GFP-MACF43 puncta analysis, neurons were nucleofected with $2 \mu \mathrm{g}$ of plasmid DNA using a mouse neuron nucleofector kit (Lonza) and Nucleofector 2b Device (Lonza), then plated normally.

\section{GFP-MACF43 and organelle transport assays}

Primary cortical neurons nucleofected with GFPMACF43 plasmid DNA were imaged at day in vitro (DIV) 1 to estimate the number of microtubule (+)-ends per $\mu \mathrm{m}$ of axon. Cells were placed in a $37^{\circ} \mathrm{C}$ imaging chamber and imaged using a $40 \times$ oil objective on a Zeiss 780 confocal microscope using ZEN imaging software (Zeiss). GFPMACF43 images were acquired every $2 \mathrm{~s}$ for a total duration of 2 min. Multiple 2-min videos were acquired for each neuron imaged, and data were used to generate a cell average, which was reported. Lysosomal and mitochondrial trafficking was assessed in cortical neurons at DIV7. Cortical neurons were incubated in either LysoTracker Red DND-99 or MitoTracker Red FM (Invitrogen) dye for $20 \mathrm{~min}$ at $37^{\circ} \mathrm{C}$, after which normal culture media was replaced and cells were transferred to a $37^{\circ} \mathrm{C}$ imaging chamber and imaged on either a Zeiss 780 confocal (MitoTracker) or a Nikon Ti-E microscope equipped with a 1.3 NA 40× CFI60 
Plan Fluor objective, piezo electric stage (Physik Instrumente), spinning disk confocal scanner unit (CSU10; Yokogawa), $488 \mathrm{~nm}$ and $561 \mathrm{~nm}$ lasers (Agilent Technologies), and an EMCCD camera (iXon Ultra 897; Andor Technology) using NIS Elements software (Nikon). Cells were imaged every $1 \mathrm{~s}$ or every $2 \mathrm{~s}$ for lysosomes and mitochondria, respectively.

\section{Western blotting}

Protein was isolated from brains of P0-P2 and adult mice by dounce homogenization and ultra-centrifugation, using a modified version of the protocol described by Vallee (1982). Protein concentrations were assessed using a BCA assay (Thermo), and relative concentration was determined using a Synergy $\mathrm{H} 1$ microplate reader (BioTek Instruments). For tubulin Western blotting, $0.25 \mu \mathrm{g}$ of brain lysate was loaded per lane, for all other blots $10 \mu \mathrm{g}$ of tissue lysate was used. Protein was run on 4-20\% gradient acrylamide gels (Bio-Rad Laboratories) at $150 \mathrm{mV}$ for $1 \mathrm{~h}$. Proteins were transferred to PVDF blotting membranes (Bio-Rad) in transfer buffer containing 25 $\mathrm{mm}$ Tris-base, $192 \mathrm{~mm}$ glycine, and 15\% methanol for $1 \mathrm{~h}$ at $75 \mathrm{~V}$ at $4^{\circ} \mathrm{C}$. Gels were dyed with Coomassie Blue (BioRad) post-transfer to assess transfer efficiency for each blot. Membranes were blocked in Tris-buffered saline containing $0.1 \%$ Tween 20 (TBST) with 5\% BSA for $1 \mathrm{~h}$ and incubated in primary antibody overnight at $4^{\circ} \mathrm{C}$. Primary antibodies were diluted in TBST containing $1 \%$ $\mathrm{BSA}$ at $4^{\circ} \mathrm{C}$ overnight. Blots were incubated in secondary antibodies diluted in TBST containing 0.5\% BSA with streptavidin-HRP (Bio-Rad, 1:10,000) for $1 \mathrm{~h}$ at room temperature. Primary antibodies for Western blotting were as follows: mouse anti-DM1A (Sigma-Aldrich T6199; $1: 10,000)$, rabbit anti-GAPDH (Cell Signaling 14C10; 1:1000), mouse anti-acetylated tubulin (Sigma-Aldrich T7451; 1:1000), mouse anti-tyrosinated tubulin (Sigma T9028; 1:5000), rabbit anti-detyrosinated tubulin (Abcam ab48389; 1:1000), mouse anti-polyglutamylated tubulin (GT335, Adipogen AG-20B-0020; 1:2000), and mouse antiSMI32 (BioLegend 801702; 1:500). Secondary antibodies used were HRP conjugated and diluted 1:5000 (Santa Cruz Biotechnology). Blots were developed in ECL solution (BioRad) and imaged using a ChemiDoc MP imager (Bio-Rad).

\section{RNA isolation + RTPCR}

RNA was isolated from flash-frozen mouse brains dissected from PO or adult mice using an SV Total RNA Isolation system (Promega). RNA concentration and purity were determined using a spectrophotometer, then cDNA was synthesized using the RT2 First Strand kit (QIAGEN). RT-PCR reactions were prepared with SYBR Green RTPCR Master mix (Thermo) and run with a CFX Connect Real-Time System (Bio-Rad). Samples were run in triplicate, results were analyzed in Excel. All qPCR data presented in this manuscript was normalized to expression of the housekeeping gene Cyclin A. Tuba $1 a^{N D /+}$ relative mRNA quantity was normalized to wild type in cases where mRNA expression was compared by genotype. In cases where tubulin genes were compared with one another, we calculated relative quantity of mRNA (RQ) using the equation $R Q=E \wedge_{-} \Delta \mathrm{CT}$, where $\mathrm{E}$ is the primer efficiency, calculated from running a dilution curve of each primer set. For all qRT-PCR experiments, three biological replicates were used per genotype. Primers were as follows: TUBA1A, F: ATTATGAGGAGGTTGGTGTG and R: TGTTGGAACACAATAAACATC; TUBA1B, F: CTCATTGCGTTACTTACCTC and R: TCACGCATGAT AGCAACG; TUBA1C, F: GGTATATAAGCCCTGTCCTG and R: CTCACGCATATITAGTCCTTG; and TUBA4A, F: ACTGTAATCGATGAGATCCG and R: AATACTAGGAA GCCCTGAAG.

\section{Experimental design and statistical analyses}

All experiments used experimental design statistics for random data. Band volume of all Western blottings was analyzed using Image Lab software (Bio-Rad). Organelle transport and GFP-MACF43 comet analysis, kymograph generation, and assessment of EM images was performed using publicly available plugins for FIJl software (National Institutes of Health). Maximum intensity projection images from en face NMJ synapses were analyzed for synaptic area, presynaptic and postsynaptic marker intensities using custom MATLAB software (MathWorks 2018). Cell counting was performed either using FIJI or MATLAB software. Statistical analyses were performed, and graphs were created using Prism version 8.0 (GraphPad). Most graphs display all data points to accurately represent the variability in each dataset, except in cases where such presentation obscured the conclusion. For all statistical analyses, means were considered to be significantly different if $p<0.05$. Statistical analyses used in each experiment are indicated in their respective figure legends. For all graphs mean \pm SEM was reported unless otherwise noted. Normality of each dataset was assessed using a Shapiro-Wilk test. In datasets with two groups, parametric data were analyzed using a Student's $t$ test, while non-parametric data were assessed by Mann-Whitney $U$ analysis of medians. Multiple groups were compared by one-way or two-way ANOVA and analyzed post hoc by either a Bonferroni or KruskalWallis test for parametric and non-parametric data, respectively. Detailed information on how each in-text statistical figure was reached can be found in Table 1.

\section{Results}

\section{Tuba1a is a major component of adult $\alpha$-tubulin mRNA}

During embryonic development, Tuba1a constitutes $\sim 95 \%$ of all $\alpha$-tubulin mRNA in the mouse brain (Miller et al., 1987). By comparison, postnatal Tuba1a expression is dramatically decreased, however certain brain regions including the neocortex and hippocampus retain Tuba1a expression into adulthood (Bamji and Miller, 1996). Many studies have focused on the role of Tuba1a during brain development, but it is unclear how much Tuba1a contributes to the $\alpha$-tubulin pool in adult neurons of the brain and spinal cord. Although TUBA1A is ubiquitously expressed in postmitotic neurons, the relative abundance of different $\alpha$-tubulin isotypes in specific neuronal subtypes over time has not been well characterized. To understand how the neuronal $\alpha$-tubulin isotype blend changes over time, we assessed mRNA for Tuba1a, Tuba1b, Tuba1c, and Tuba4a in the cortex and spinal cord of post-natal day 0 (P0) and adult wild- 
A
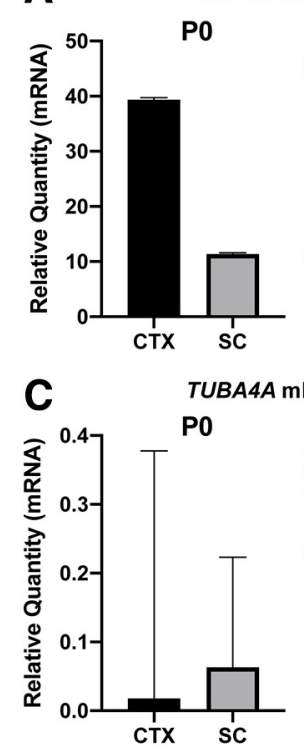

B
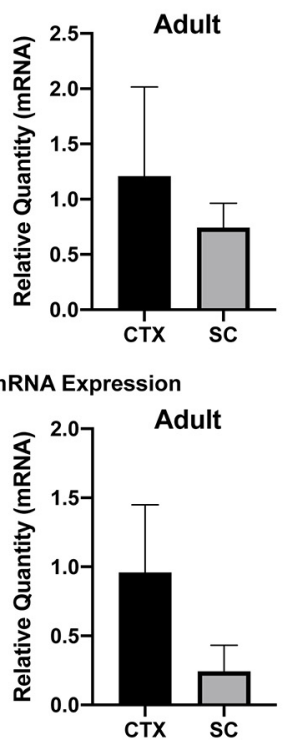

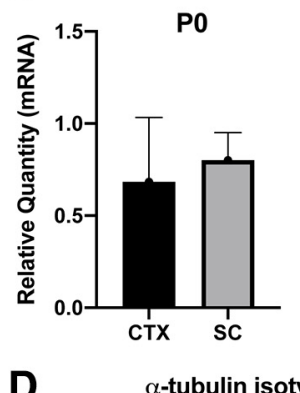

D $\alpha$-tubulin isotype ratio

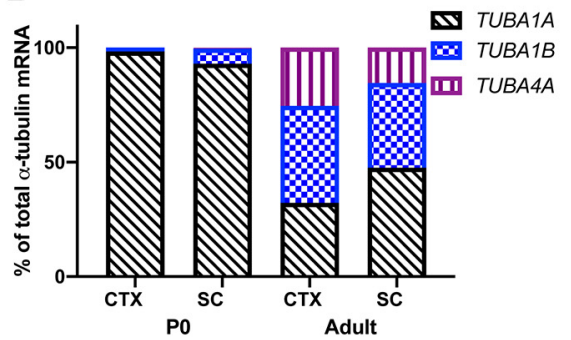

Figure 1. Changes in $\alpha$-tubulin isotype expression in cortex (CTX) and spinal cord (SC) between post-natal day 0 (P0) and adult. $\boldsymbol{A}$, Bar graphs representing relative quantity of TUBA1A mRNA in P0 (left) and adult (right) mouse cortex and spinal cord. B, Bar graph representing relative quantity of TUBA1B mRNA in cortex and spinal cord of P0 (left) and adult (right) mice. $\boldsymbol{C}$, Bar graph representing relative quantity of TUBA4A mRNA in cortex and spinal cord of P0 (left) and adult (right) mice. $\boldsymbol{D}$, Bar graphs representing the $\alpha$-tubulin isotype mRNA expression data from $\boldsymbol{A}-\boldsymbol{C}$ as a percentage of the total $\alpha$-tubulin isotype composition. Three animals per genotype were analyzed for each time point, with three technical replicates per animal. Data are plotted as relative mRNA quantity normalized to housekeeping gene Cyclin A. Bars represent mean \pm SEM.

type mice (Fig. 1A-C; $N=3$ ). Unfortunately, levels of Tuba1c mRNA were too low to be reliably detected in our assays, thus we have omitted those data. Although the relative quantity of mRNA for each isotype differed between the cortex and spinal cord, we found that the ratio of $\alpha$-tubulin isotypes was comparable between spinal cord and cortex, in both newborn and adult animals (Fig. $1 D ; N=3$ ). Intriguingly, although Tuba1a expression is downregulated between development and adulthood, we found that in wild-type adult mice, Tuba1a comprises $32 \%$ of all cortical and $47 \%$ of all spinal cord $\alpha$-tubulin mRNA (Fig. 1D). These data demonstrate that although Tuba1a mRNA abundance decreases postnatally, Tuba1a is the most abundant $\alpha$-tubulin isotype in adult spinal cord and the second-most abundant $\alpha$-tubulin in adult cortex. Because Tuba1a constitutes a large percentage of the total $\alpha$-tubulin in both cortical and spinal neurons over time, we predict that Tuba1a plays an important role in the adult neuronal microtubule network.

\section{Tuba1a $^{N D}$ is a loss-of-function mutation in mice}

While it is known that Tuba1a contributes significantly to development, it has been difficult to determine how this isotype contributes to adult neuronal function due to the lack of available tools to study Tuba1a protein. Previous data in yeast (Gartz Hanson et al., 2016) suggested that heterozygous Tuba1a ${ }^{N D /+}$ mice may have reduced Tuba1a function. To determine whether the Tuba1a ND substitution affects levels of $\alpha$-tubulin in mice, we isolated protein from wild-type and Tuba1a $a^{N D /+}$ brains at P0-P2. We did not include homozygous Tuba1a ${ }^{N D / N D}$ mouse brain lysates due to their perinatal lethality. Quantitative Western blottings revealed an approximate $50 \%$ decrease in $\alpha$-tubulin protein in heterozygous Tuba1 $a^{N D /+}$ mouse brains compared with wild-type at birth (Fig. $2 A, B ; N=4$, $p<0.0001)$ consistent with the molecular impact of the ND substitution in yeast reported in Hanson et al., (2016). This reduction in $\alpha$-tubulin protein was not due to a change in brain size, as weights between wild-type and Tuba1a ${ }^{N D /+}$ brains were comparable (Extended Data Fig. $2-1 ; N=10, p=0.68)$. Adult Tuba1a ${ }^{N D /+} \alpha$-tubulin protein levels were comparable to wild type (Fig. $2 A, B ; N=6$, $p=0.38$ ). The observed reduction in developmental $\alpha$-tubulin indicates that the availability of $\alpha$-tubulin protein is decreased in Tuba1a ${ }^{N D /+}$ neurons during a critical period for neuronal microtubule network establishment. As total $\alpha$-tubulin protein levels normalized in the brains of adult Tuba1 ${ }^{N D /+}$ mice, we assessed brain tubulin PTMs because PTMs influence binding interactions with MAPs and overall microtubule function. Western blottings of whole-brain lysates from adult wild-type and Tuba $1 \mathrm{a}^{\mathrm{ND} /+}$ mice revealed no genotypic difference in the abundance of acetylated $(N=5 ; p=0.66)$, tyrosinated $(N=3 ; p=0.52)$, detyrosinated $(N=5 ; p=0.81)$, or polyglutamylated $(N=3 ; p=0.31) \alpha$-tubulin relative to the total $\alpha$-tubulin (Fig. 2C,D). Therefore, we conclude that Tuba $1 a^{N D}$ substitution reduces the availability of $\alpha$-tubulin protein developmentally, but does not impact the PTM landscape of adult brain microtubules.

Mice have nine $\alpha$-tubulin genes and mammalian neurons express at least four distinct $\alpha$-tubulin genes (Miller et al., 1987; Bamji and Miller, 1996; Gloster et al., 1999; Zhang et al., 2014). Mutations that disrupt the major isoform, Tuba1a, could potentially induce compensatory changes to the expression of other $\alpha$-tubulin mRNAs. We 


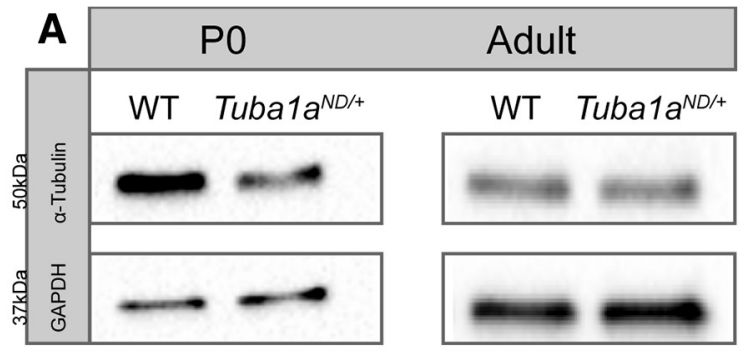

B Total $\alpha$-Tubulin
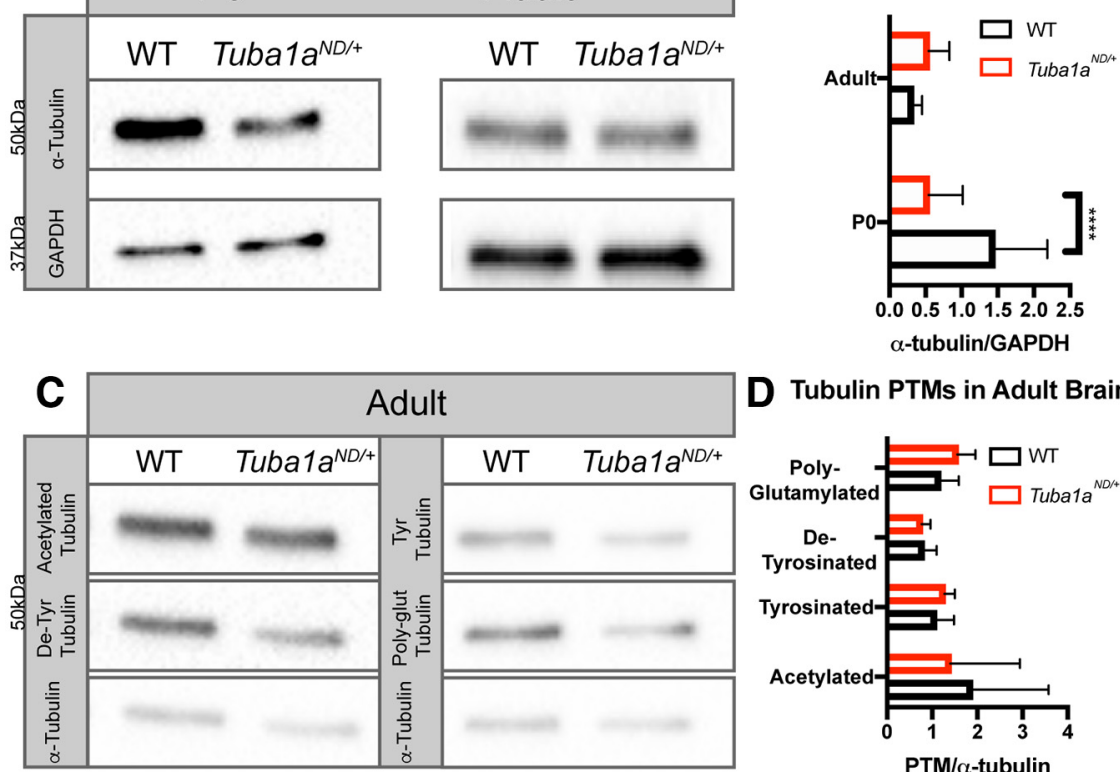

D Tubulin PTMs in Adult Brain
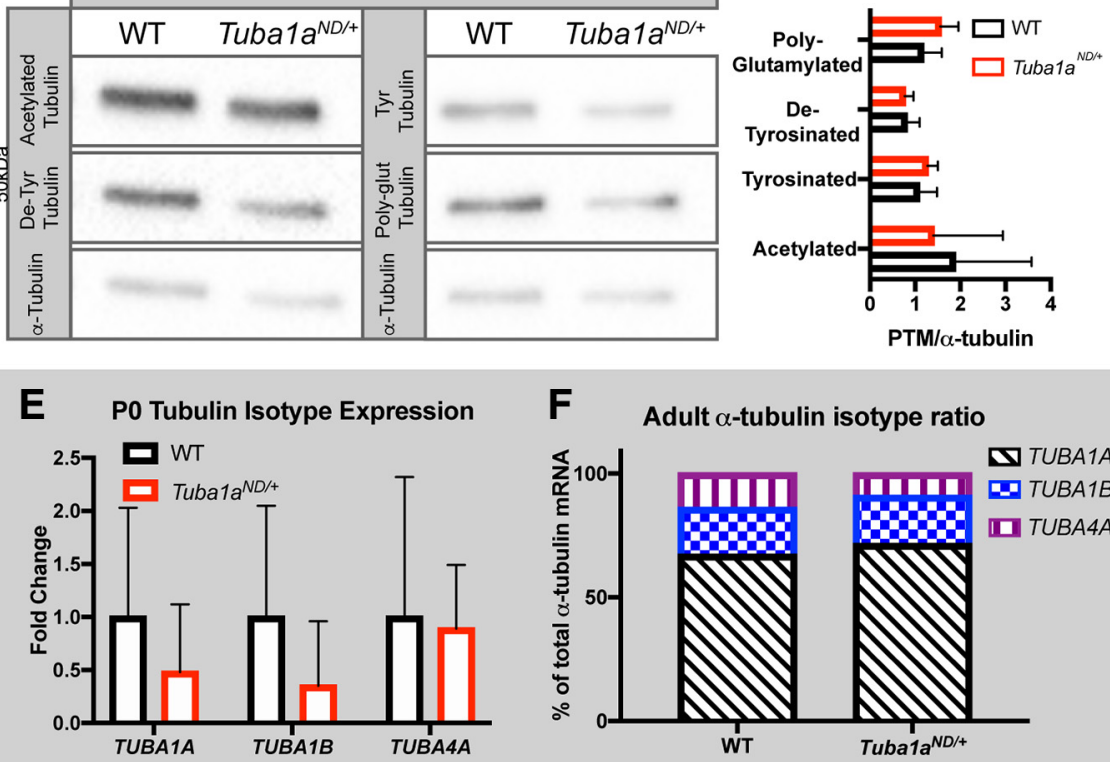

F Adult $\alpha$-tubulin isotype ratio

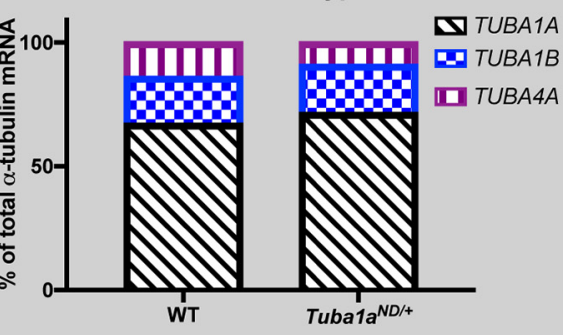

G Adult Tubulin Isotype Expression
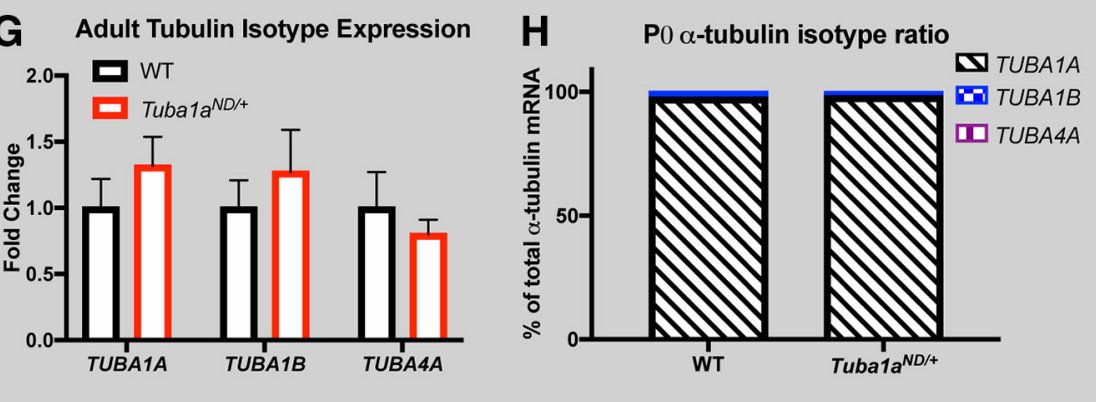

Figure 2. $\alpha$-Tubulin protein is decreased in P0 Tuba1 $a^{N D /+}$ brains. $\boldsymbol{A}$, Western blottings representing $\alpha$-tubulin abundance in P0 (left) and adult (right) Tuba1a ${ }^{N D /+}$ and wild-type (WT) mouse brains. B, Bar graph quantifying $\alpha$-tubulin protein in P0 and adult brain tissue lysates relative to GAPDH ( $N=3$ mice, $p<0.0001$ by two-way ANOVA). Quantifications are representative of at least three separate experiments. $\boldsymbol{C}$, Western blottings showing the abundance of $\alpha$-tubulin PTMs acetylation (top left), tyrosination (top right), detyrosination (center left), and polyglutamylation (center right), and total $\alpha$-tubulin (bottom) in adult wild-type and Tuba1a ${ }^{N D /+}$ whole-brain lysates. $\boldsymbol{D}$, Bar graph quantification of Western blotting data shown in $\boldsymbol{C}$. PTM band volume was normalized to $\alpha$-tubulin $(\boldsymbol{p}>0.05$ for all by $t$ test). $\boldsymbol{E}, \boldsymbol{F}$, mRNA expression of brain $\alpha$-tubulin isotypes in Tuba1a ${ }^{N D /+}$ and wild-type mice at P0. Data are plotted as fold change in Tuba1a $a^{N D /+}$ relative to wild type (E) or as a percentage of the total $\alpha$-tubulin isotype composition $(\boldsymbol{F} ; p>0.05$ for all by two-way ANOVA). $\boldsymbol{G}, \boldsymbol{H}$, Bar graph representing mRNA expression of brain $\alpha$-tubulin isotypes in adult Tuba1 $a^{N D /+}$ and wild-type mice. Data are plotted as fold change in Tuba1a $a^{N D /+}$ relative to wild type $(\mathbf{G})$ or as a percentage of the total $\alpha$-tubulin isotype composition $(\boldsymbol{H} ; p>0.05$ for all by two-way ANOVA). All Western blotting data represents three replicate blots with lysates from at least three animals per genotype. Bars represent mean \pm SEM. $* * * * p<0.0001$.

found that mRNA expression of Tuba1a and other brain $\alpha$-tubulin isotypes was not significantly changed in heterozygous Tuba1a ${ }^{N D /+}$ P0 brain lysates (Fig. 2E; $p>0.9$ for all comparisons by two-way ANOVA). Additionally, the relative ratio of each $\alpha$-tubulin isotype mRNA was unchanged in Tuba1 $a^{N D /+}$ P0 brain lysates, with Tuba1a constituting $\sim 98 \%$ of all $\alpha$-tubulin mRNA in both wild-type and Tuba1a ${ }^{N D /+}$ brains (Fig. 2F; $N=3, p=0.92$ ). We conclude 


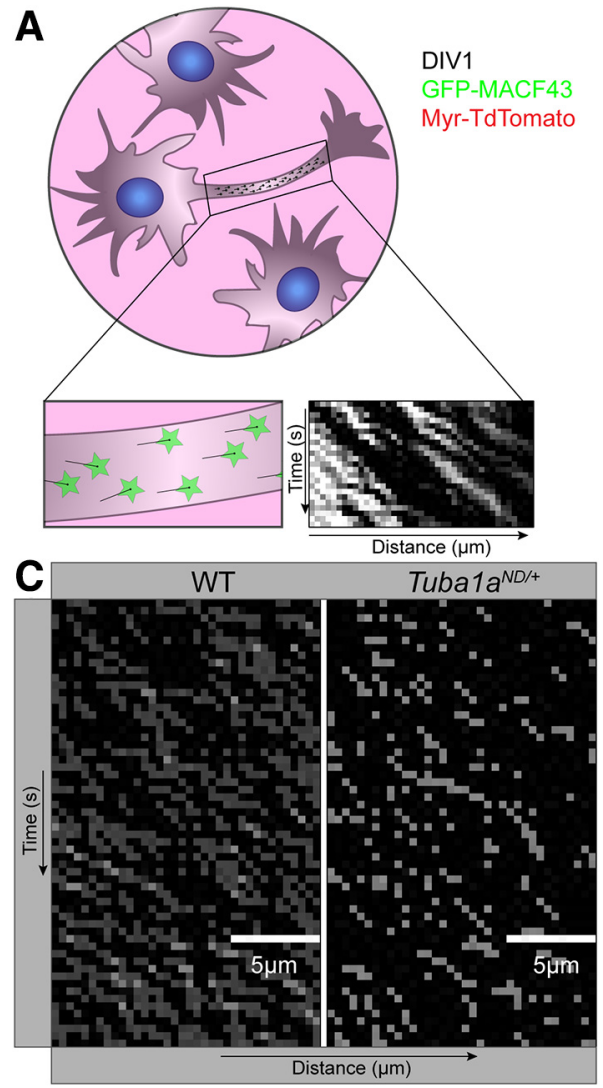

B

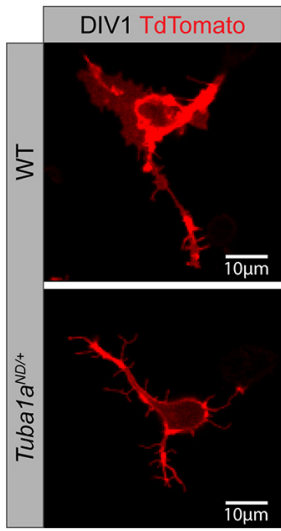

D

Growing Microtubule Ends/um

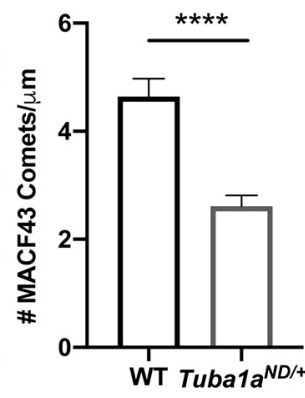

Figure 3. Reduced TUBA1A function results in fewer microtubule tracks in neurites. $\boldsymbol{A}$, Schematic of experimental design, illustrating microtubule plus-end tracker, GFP-MACF43, puncta analysis and kymograph generation. $\boldsymbol{B}$, Representative images of day in vitro (DIV) 1 wild-type (WT) and Tuba1a ${ }^{N D /+}$ neurons, visualized using a membrane-bound Myr-TdTomato. Scale bars: $10 \mu \mathrm{m}$. $\boldsymbol{C}$, Representative kymograph plots generated from GFP-MACF43 images in wild-type (left) and Tuba1a ${ }^{N D /+}$ (right) neurons. Scale bars: $5 \mu \mathrm{m}$. $\boldsymbol{D}$, Bar graph representing the number of GFP-MACF43 puncta per $\mu \mathrm{m}$ of neurite in DIV1 wild-type and Tuba1a ${ }^{N D /+}$ cortical neurons. Bars illustrate mean density of GFP-MACF43 puncta per kymograph and error bars indicate SEM ( $n=24$ neurons, $p<0.0001)$. Statistical differences between groups were assessed by $t$ test. $* * * * p<0.0001$.

from these results that alternative $\alpha$-tubulin isotypes are not upregulated to compensate for the loss of TUBA1A protein in the developing brain. We examined the abundance of $\alpha$-tubulin isotype mRNAs in the cortex of adult wild-type and Tuba1 ${ }^{N D /+}$ mice and found that Tuba1a remains one of the predominant $\alpha$-tubulins for both genotypes (Fig. 2G; $N=3 ; p>0.7$ for all by two-way ANOVA). The modest, but statistically insignificant increase in Tuba1a mRNA in the adult Tuba1a ${ }^{N D /+}$ cortex slightly shifted the ratio of $\alpha$-tubulin isotype mRNAs in the adult cortex, with an increased percentage of Tuba1a $\alpha$-tubulin mRNA compared with the other isotypes (Fig. 2H; $p<0.0001$ in Tuba1 $a^{N D /+}$ cortex compared with wild-type by two-way ANOVA). Together, these data demonstrate that Tuba $1 a^{N D /+}$ transiently depletes $\alpha$-tubulin protein during a critical developmental window, without inducing compensatory upregulation of other $\alpha$-tubulin isotypes.

\section{Reduced TUBA1A function results in fewer microtubule tracks in neurites}

To determine how reduced TUBA1A impacts the development of a neuronal microtubule network, we used primary neurons from wild-type and Tuba1a ${ }^{N D /+} \mathrm{PO}-\mathrm{P} 2$ cortices to examine microtubule organization in vitro. Wildtype and Tuba $1 \mathrm{a}^{\mathrm{ND} /+}$ primary cortical neurons were nucleofected with membrane-bound Myr-TdTomato and GFPMACF43, which binds to polymerizing microtubule $(+)$-end-binding proteins (EBs) without affecting microtubule function (Yau et al., 2016). Kymograph plots generated from GFP-MACF43 videos allowed us to interrogate the number of growing microtubule $(+)$-ends per micrometer of neurite at day in vitro (DIV) 1 (Fig. $3 A-C$ ). We found that Tuba $1 a^{N D /+}$ neurites have approximately half the number of GFP-MACF43 puncta per micrometer of neurite as wild-type neurites $[2.610 \pm 0.20$ growing microtubule ends in Tuba $1 a^{N D /+}$ vs $4.636 \pm 0.34$ in wild type (Fig. $3 D$ ); $n=24$ neurites; $p<0.0001]$. These data suggest the approximate $50 \%$ reduction in $\alpha$-tubulin protein in observed in developmental Tuba1 $\mathrm{a}^{\mathrm{ND} /+}$ mice causes functional loss of microtubule track density within developing Tuba1 $a^{N D /+}$ neurons.

\section{TUBA1A is required for functional intracellular transport}

One critical function of neuronal microtubules is to facilitate intracellular trafficking of proteins, mRNAs, and organelles between the cell body and distal neurites. Thus, 

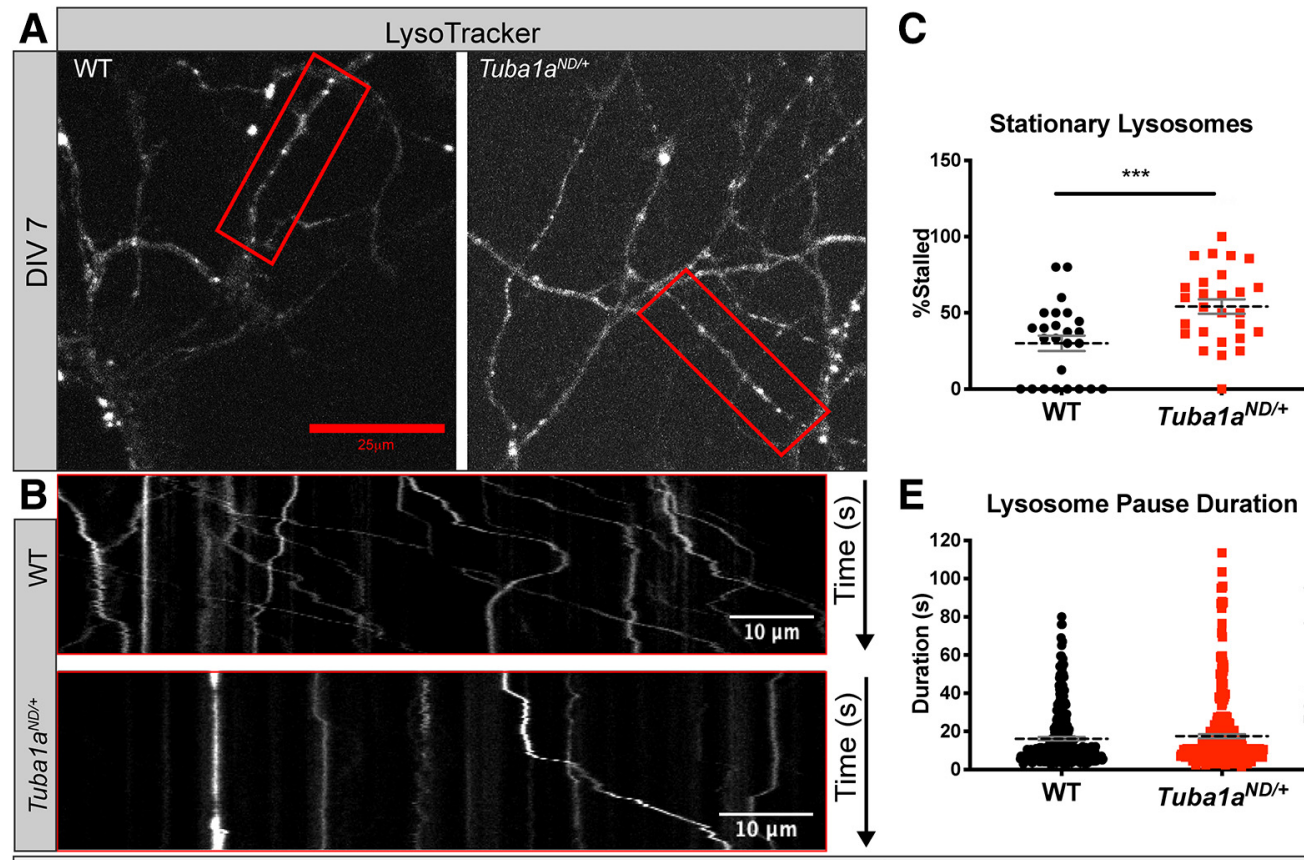

D
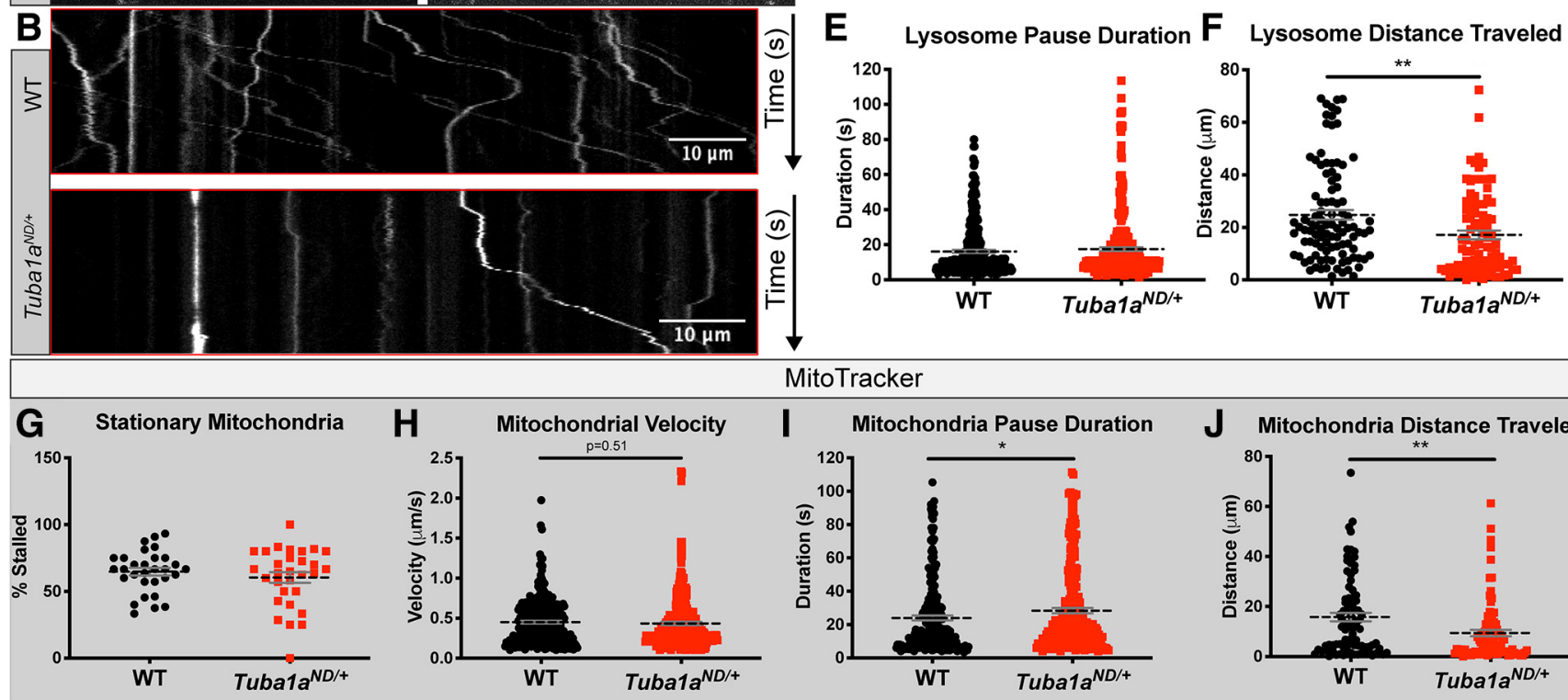

MitoTracker
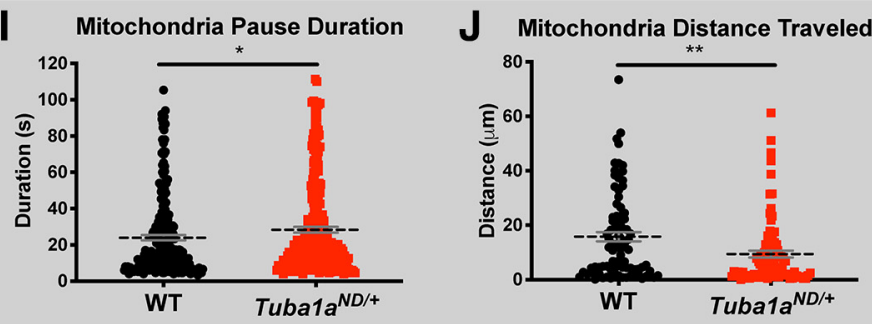

Figure 4. Tuba1 $\mathrm{a}^{\mathrm{ND/+}}$ neurons have deficits in organelle transport caused by increased stationary cargoes. $\boldsymbol{A}$, Still images from time-lapse microscopy of DIV3 cortical neurons labeled with LysoTracker dye to mark lysosomes in wild-type (WT) and Tuba1a ${ }^{\text {ND/+ }}$ neurons. B, Representative kymograph plots of lysosome movement over time within select neurites for wild type (top) and Tuba1a ${ }^{N D /+}$ (bottom). C , Scatter plot representing the percent of stationary lysosomes per kymograph in wild-type and Tuba1a ${ }^{N D /+}$ neurons. Data points represent individual neurons $(N=3$ mice, $n=25$ neurons, $p=0.001)$. $\boldsymbol{D}$, Scatter plot representing lysosome velocities in $\mu \mathrm{m} / \mathrm{s}$ for wild-type and Tuba1a ${ }^{N D /+}$ neurons. Data points represent individual lysosomes $(n=635$ lysosomes, $p=0.27)$. $\boldsymbol{E}$, Scatter plot representing pause duration (s), for moving lysosomes in wild-type and Tuba1a ${ }^{N D /+}$ neurons. Data points represent individual pause events $(n=338$ events, $p=0.56)$. $\boldsymbol{F}$, Scatter plot representing total distance traveled ( $\mu \mathrm{m})$ by moving lysosomes in wildtype and Tuba1a ${ }^{N D /+}$ neurons. Data points represent individual lysosomes $(n=87, p=0.003)$. $G$, Scatter plot representing the percentage of stationary mitochondria in wild-type and Tuba1 $a^{N D /+}$ neurons. Data points represent individual neurons $(N=3$ mice, $n=30$ neurons, $p=0.34$ by). $\boldsymbol{H}$, Scatter plot the velocity of short mitochondrial movements in wild-type and Tuba1a ${ }^{N D /+}$ neurons. Data points represent individual mitochondria $(N=3$ mice, $n=301$ mitochondria, $p=0.45)$. I, Scatter plot representing pause duration (s), for moving mitochondria in wild-type and Tuba1a ${ }^{N D /+}$ neurons. Data points represent individual pause events $(n=417$ events, $p=0.79)$. $\boldsymbol{J}$, Scatter plot representing total distance traveled $(\mu \mathrm{m})$ by moving mitochondria in wild-type and Tuba1a $\mathrm{N}^{\mathrm{N} /+}$ neurons. Data points represent individual mitochondria $(n=86, p=0.01)$. Cortical neurons from three animals per genotype were analyzed. Lysosomes were imaged once every second for $3 \mathrm{~min}$, mitochondria were imaged once every $2 \mathrm{~s}$ for 2 min. Statistical differences between groups were assessed by Student's $t$ test; $* * p<0.01 ; * * * p<0.001$. In all plots, line designates mean with error bars indicating SEM. $* p<0.05 ; * * p<0.01 ; * * * p<0.001$.

we explored the possibility that reduced TUBA1A function and a reduced density of microtubule tracks impairs microtubule-based intracellular transport. To evaluate how loss of TUBA1A impacts the dynamics of microtubulebased transport, we examined transport of lysosomes in cultured primary neurons from P0-P2 wild-type and Tuba1a ${ }^{N D /+}$ cortices.

Lysosomes facilitate enzymatic degradation of intracellular components and are highly motile organelles that are transported bidirectionally on microtubules through all compartments of the neuron, making them an ideal organelle in which we could examine microtubule-based transport efficiency. Time-lapse images of lysosome movement in wild-type or Tuba $1 a^{N D /+}$ cortical neurons were used to generate kymograph plots of lysosome movement over time within single wild-type and Tuba1 $\mathrm{a}^{N D /+}$ axons and dendrites (Fig. $4 A, B$ ), from which we could measure velocity, dynamics of movement, and total distance traveled. From these analyses we found that during the 3 -min imaging period, $\sim 70 \%$ of wild-type 
lysosomes were moving, while the remainder were stationary for the duration of the video (Fig. $4 C ; N=3$ mice, $n=25$ neurons). A significantly higher number of Tuba $1 a^{N D /+}$ lysosomes were stationary compared with wild type, with only $46 \%$ of Tuba $1 a^{N D /+}$ lysosomes moving during the $3-\mathrm{min}$ window (Fig. $4 C ; p=0.001$ ). Of those lysosomes that were moving, the velocity of each movement was unchanged between wild-type and Tuba1 $\mathrm{a}^{\mathrm{ND/+}}$ neurons, with average velocity of $0.56 \pm 0.02$ and $0.53 \pm$ $0.02 \mu \mathrm{m} / \mathrm{s}$ for wild-type and Tuba $1 a^{N D /+}$ neurons, respectively (Fig. $4 D ; n=635$ lysosomes, $p=0.27$ ). During active transport, cargoes exhibit phases of movement and pausing, thus we wanted to evaluate the dynamics of lysosome movement by measuring pause duration for moving lysosomes. There was no difference in pause duration of moving lysosomes in Tuba1a ${ }^{N D /+}$ neurons, which paused on average $17.51 \pm 1.0 \mathrm{~s}$, compared with a $16.19 \pm 0.81-$ $\mathrm{s}$ pause duration in wild-type neurons (Fig. $4 E ; n=337$ events, $p=0.56)$. Finally, we observed a significant decrease in the total distance traveled by lysosomes over the 3-min imaging window, from $24.77 \pm 1.84 \mu \mathrm{m}$ in wildtype neurons to $17.19 \pm 1.66 \mu \mathrm{m}$ in Tuba1 $a^{\mathrm{ND/+}}$ neurons (Fig. 4F; $n=100$ neurons, $p=0.003$ ). These data demonstrate that Tuba1a $\mathrm{ND/+}^{\mathrm{N}}$ substitution causes a deficit in neuronal intracellular transport by which Tuba1a ${ }^{N D /+}$ lysosomes do not travel as far as wild type within the same time window.

To further understand the impact of reduced TUBA1A function on neuronal microtubule-based transport, we examined mitochondrial trafficking in cultured primary Tuba1a $a^{N D /+}$ neurons compared with wild type. Transport of mitochondria and lysosomes is conducted by a different set of kinesins (Hurd and Saxton, 1996; Tanaka et al., 1998; Hendricks et al., 2010; Maday et al., 2014). Therefore, examining transport of multiple cargoes provides mechanistic insight into the intracellular trafficking disruption in Tuba1a $a^{N D /+}$ neurons. The analyses described above were repeated in DIV7 wild-type and Tuba1a $a^{N D /+}$ cortical neurons that had mitochondria labeled (Extended Data Fig. 4-1). Over the course of a 2min imaging period, we observed that the number of moving mitochondria was similar between wild-type (36\%) and Tuba $1 a^{N D /+}(40 \%)$ neurons, and the remainder were stationary for the duration of the video (Fig. 4G; $N=3$ mice, $n=30$ neurons, $p=0.38)$. Importantly, the relative number of stationary mitochondria was more than double the number of stationary lysosomes in wild-type neurons $(64.78 \pm 2.9 \%$ and $30.01 \pm 5.0 \%$, respectively; Fig. $4 C$, G). Similar to what was observed for lysosomal trafficking, we found that the velocity of moving mitochondria was unchanged between wild-type $(0.45 \pm 0.02 \mu \mathrm{m} / \mathrm{s})$ and Tuba1a ${ }^{N D /+}(0.43 \pm 0.02 \mu \mathrm{m} / \mathrm{s})$ neurons (Fig. $4 H ; n=301$ events, $p=0.51$ ). These mitochondrial movements were interrupted by differential periods of pause, with a significant increase in Tuba $1 a^{N D /+}$ mitochondrial pausing compared with wild type, with average pause durations of $28.36 \pm 1.6$ and $24 \pm 1.4 \mathrm{~s}$, respectively (Fig. $4 / ; n=251$ events, $p=0.02$ ). The total distance traveled by motile mitochondria during the 2-min imaging window was found to be significantly decreased in Tuba $1 a^{N D /+}$ neurons, with
Tuba1a $a^{N D /+}$ mitochondria traveling $9.46 \pm 1.3 \mu \mathrm{m}$ on average compared with $15.8 \pm 1.7 \mu \mathrm{m}$ in wild-type neurons (Fig. $4 \mathrm{~J} ; n=301$ events, $p=0.01$ ). Combined, these analyses indicate that a TUBA1A deficit impairs transport of multiple organelle types in cortical neurons, resulting in a significant reduction in the total distance of organelle movement.

\section{Diminished TUBA1A causes late-onset behavioral deficits}

Having established that neurons from young Tuba1a ${ }^{N D}$ mutant animals exhibit deficits in microtubule density and organelle trafficking, we next asked whether these lead to impairments at the level of behavior. The Tuba1a ${ }^{N D}$ mutant allele was identified due to locomotor deficits apparent in homozygous mutant embryos. Tuba1 ${ }^{N D / N D}$ homozygous substitution induced severe neurodevelopmental abnormalities that impaired motor neuron function and caused perinatal lethality (Gartz Hanson et al., 2016). In contrast, Tuba 1a $\mathrm{ND}^{\mathrm{N} /+}$ embryos survive to adulthood and were indistinguishable from wild-type siblings at the age of weaning (P21). Tuba1a ${ }^{N D /+}$ embryos exhibit normal presynaptic and postsynaptic motor development within the diaphragm and respond to touch stimulation (Gartz Hanson et al., 2016). However, as Tuba $1 a^{N D /+}$ mice aged, we noticed differences in locomotion that were quantified with gait analysis. While the gait of two-month-old Tuba1 $\mathrm{a}^{\mathrm{NDT+}}$ mice is not significantly different from wild type $(N=2)$, by three months of age, Tuba1a ${ }^{N D /+}$ mice develop an ataxic gait, as evidenced by a wider rear stance (Fig. $5 A, B ; N=5$, $p=0.02)$. Tuba1a ${ }^{N D /+}$ mice developed impaired motor coordination by five months of age, as assessed by rotarod performance (Fig. 5C; $N=10, p=0.03$ ). After the initial onset of behavioral phenotypes, Tuba $1 a^{N D /+}$ mice performed worse than wild type at all time points examined $\left(p<0.05\right.$ for all). Heterozygous Tuba1a ${ }^{N D /+}$ mice did not display deficits in motor learning as assessed by the difference in rotarod performance between trials (Fig. 5D; $N=10$, wild-type $R^{2}=0.98$, Tuba1a ${ }^{N D /+} R^{2}=0.89, p=0.31$ by linear regression). Locomotor impairment of these mice impeded more rigorous testing of cognitive function. Importantly, forelimb function, and all other parameters of gait were similar to that measured in wild-type animals (Extended Data Fig. 5-1). Body weights of Tuba1a ${ }^{N D /+}$ mice were comparable to that of control mice (Fig. 5F; $N=7, p=0.24$ ), and forelimb muscular strength was not impaired (Fig. $5 E ; N=7, p=0.19$ ). These results indicate that neither altered muscle content nor gross muscle function is responsible for the observed motor deficits, indicating Tuba1a $a^{N D}$-induced neuronal dysfunction is a likely cause. The hindlimb-specificity of this behavioral phenotype further suggests that neurons with extremely long axons, such as those that innervate the hindlimbs, may be exquisitely sensitive to perturbations of Tuba1a.

Tuba1a is expressed in all postmitotic neurons (Paden et al., 1995; Bamji and Miller, 1996), therefore we predicted that behavioral impairments induced by Tuba1 $\mathrm{a}^{\mathrm{ND/+}}$ substitution would not be restricted to motor neurons. To assess whether Tuba1 $a^{N D /+}$ adult mice had deficits in sensory behavior, we examined hind-paw sensory 
A

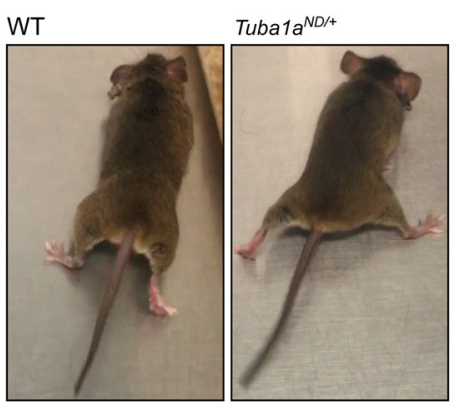

C

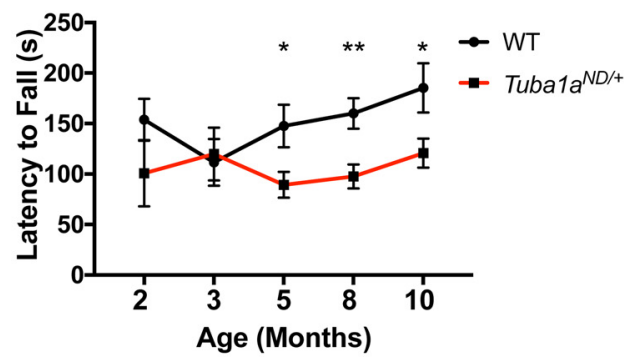

E

Front Grip Strength

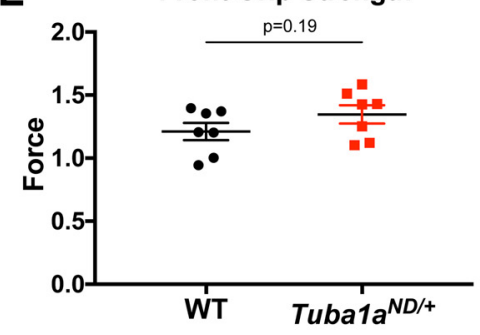

G

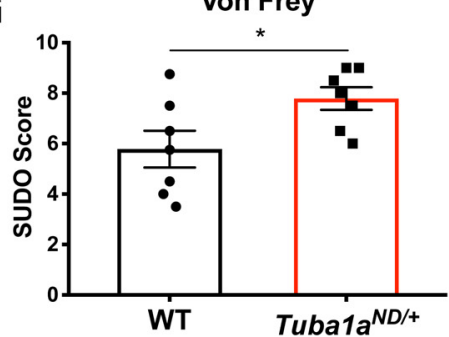

B

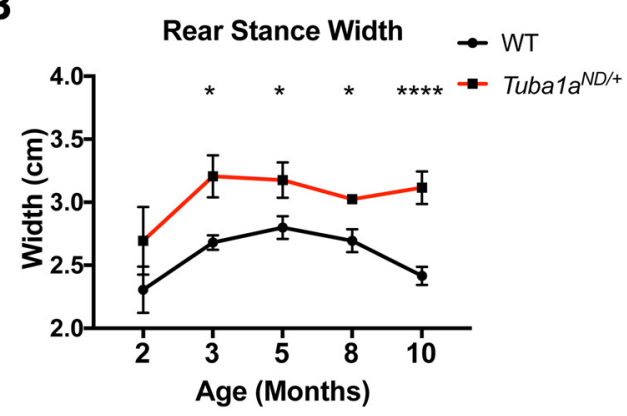

D

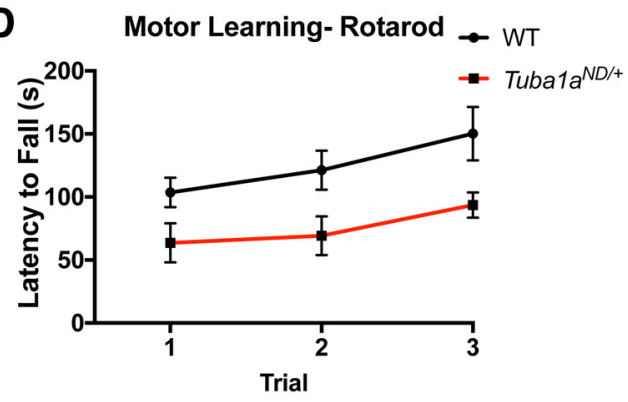

$\mathbf{F}$

Body Weight

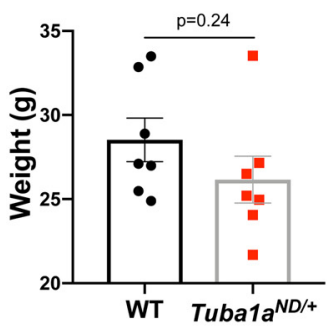

H Thermal Pain Sensitivity

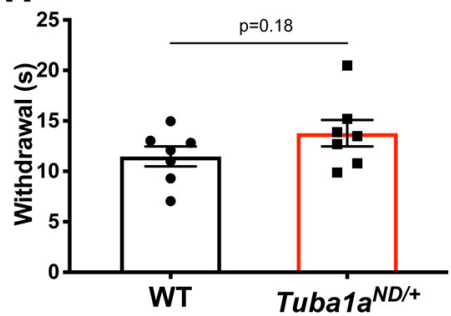

Figure 5. Tuba1a $\mathrm{ND/}^{\mathrm{N}}$ mice exhibit adult-onset motor and sensory behavioral deficits. $\boldsymbol{A}$, Images depicting gait abnormalities in Tuba1a $a^{N D /+}$ adult mice compared with wild-type (WT). Still images were taken from video of Tuba1a ${ }^{N D /+}$ and wild-type mice midstride. $\boldsymbol{B}$, Line graph quantifying changes in rear stance width at two months $(p=0.61)$, three months $(p=0.02)$, five months $(p=0.04)$, eight months $(p=0.03)$, and 10 months $(p<0.0001)$ of age in wild-type and Tuba1 $a^{N D /+}$ mice. $C$, Line graph representing latency to fall on rotarod task at two months $(p=0.31)$, three months $(p=0.82)$, five months $(p=0.03)$, eight months $(p=0.004)$, and 10 months $(p=0.04)$ of age in wild-type and Tuba1 $a^{N D /+}$ mice. Points depict the mean stance width or latency to fall by genotype with SEM. The same mice were analyzed throughout, and a two-way ANOVA was used to assess statistical significance between groups. Number of mice per genotype is as follows: $N=2$ at two months, $N=5$ at three months, $N=8$ at five months, $N=12$ at eight months, $N=8$ at 10 months. $\boldsymbol{D}$, Line graph representing performance over three subsequent rotarod trials in a single testing session at five months of age for Tuba1a ${ }^{N D /+}$ compared with wild type. Slopes of the lines for wild type and Tuba1 $a^{N D /+}$ were compared by linear regression and were not found to be significantly different $\left(p=0.32\right.$, wild-type $R^{2}=0.98$, Tuba1 $\left.a^{N D /+} R^{2}=0.89\right)$. $E$, Scatter plot of grip strength assessed in the forelimbs of female five-month-old wild-type and Tuba1 ${ }^{N D /+}$ mice $(N=7, p=0.19)$. $\boldsymbol{F}$, Bar graph of total body weight for eight- to 10-month-old Tuba1a ${ }^{N D /+}$ and wild-type female mice $(N=7, p=0.24)$. G. Bar graph of SUDO scores Bonin et al. (2014) for Von Frey mechanical sensation testing in three- to six-month-old wild-type and Tuba1 $a^{N D /+}$ mice $(N=7, p=0.04)$. $\boldsymbol{H}$, Bar graph of time to withdraw from thermal stimulus in Hargreaves behavioral analysis on three-month-old wild-type and Tuba1a $a^{N D /+}$ mice $(N=7$, $p=0.19)$. Sex as a variable did not significantly impact rear stance width or rotarod performance at any time point examined $(N=7$ females, $N=5$ males; $p>0.05$ for all comparisons of male vs female). Sex was also not found to significantly influence sensory behavior $(N=7$ males, $N=2$ females; $p>0.05$ for all comparisons); $* p<0.05, * * p<0.01$, $* * * * p<0.0001$. For line graphs, points indicate mean by genotype at each time point \pm SEM. For Bar graphs, bars represent mean \pm SEM. $* p<0.05 ; * * p<0.01 ; * * * * p<0.0001$. 


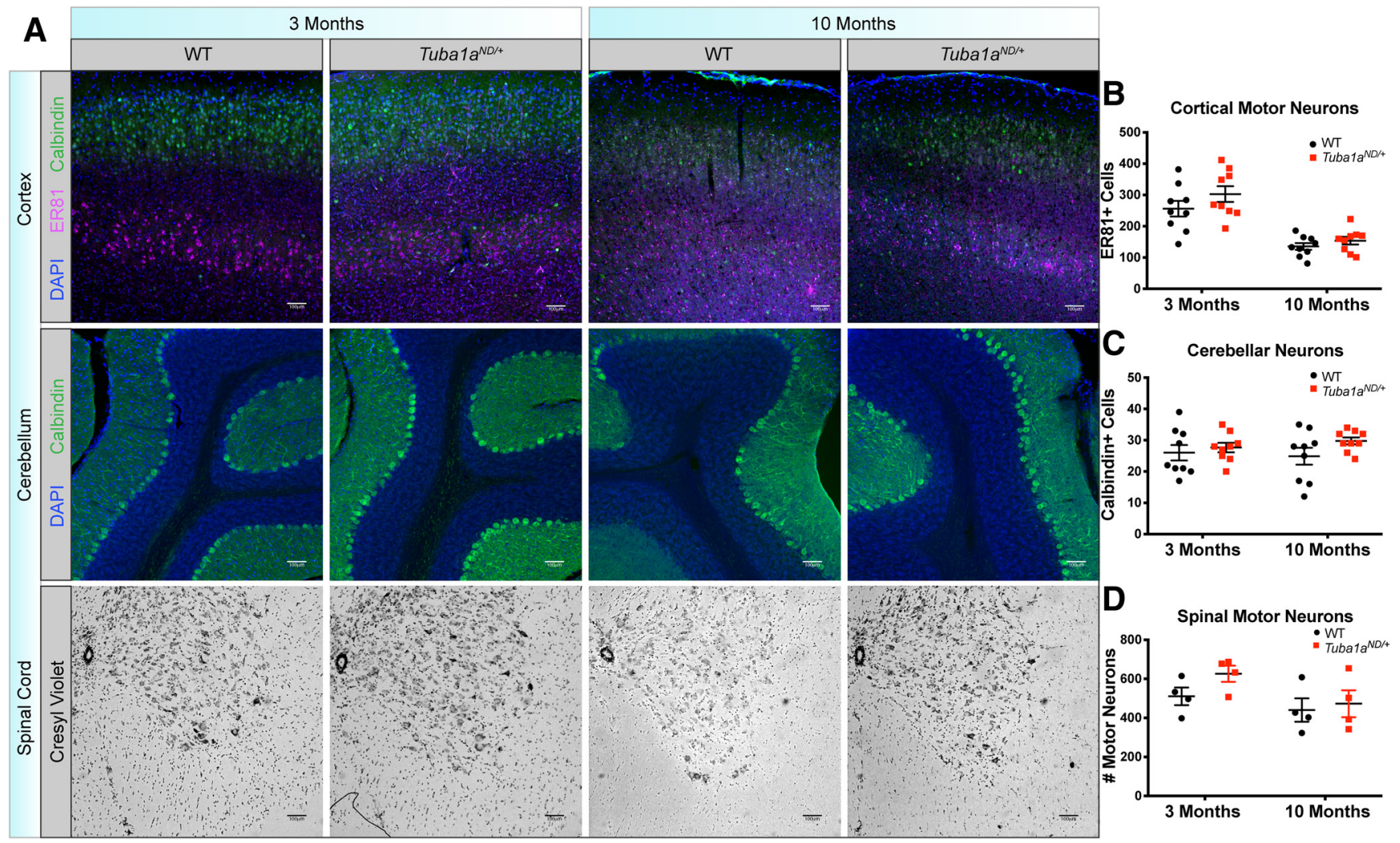

Figure 6. Tuba1 $a^{N D /+}$ does not impact neuronal cell body survival. $\boldsymbol{A}$, top row, Coronal sections of motor cortex immunolabeled with calbindin (green), ER81 (magenta), and DAPI (blue) in wild-type and Tuba1a ${ }^{N D /+}$ mice at three months (left) and 10 months (right) of age at $20 \times$ magnification. Center row, Sagittal sections of cerebellum labeled with calbindin (green) and DAPI (blue) in wild-type and Tuba1 $\mathrm{a}^{\mathrm{ND/+}}$ mice at three months (left) and 10 months (right) of age at $20 \times$ magnification. Bottom row, Nissl-stained coronal sections of the lumbar spinal cord in wild-type and Tuba1a ${ }^{N /+}$ mice at three months (left) and 10 months (right) of age at $4 \times$ magnification. $\boldsymbol{B}$, Scatter plot representing the number of ER81+ Layer $V$ neurons per image in motor cortex at three and 10 months $(N=3$ mice, $p=0.19$ and $p=0.78$, respectively). $\boldsymbol{C}$, Scatter plot representing the number of calbindin + Purkinje neurons per image in the cerebellum at three and 10 months $(N=3, p=0.94$ and $p=0.35$, respectively). $\boldsymbol{D}$, Scatter plot representing the number of Nissl+ ventral horn motor neurons per image in the spinal cord at three and 10 months $(N=3, p=0.66$ and $p=0.99$, respectively). Motor neurons were identified morphologically. Data points represent ROls with horizontal line depicting mean with SEM. Data points were nested by animal and analyzed by two-way ANOVA.

function using two separate assays in mature mice. Response to hind-paw stimulation with Von Frey filaments revealed an increased threshold for retraction in Tuba1a ${ }^{N D /+}$ compared with wild-type mice, indicating reduced sensation of mechanical stimuli (Fig. $5 G ; N=7, p=0.04$ ). Additionally, adult Tuba1a $\mathrm{N}^{\mathrm{ND/+}}$ animals displayed a modest, but not statistically significant decrease in thermal pain sensation of the hind-paws, as measured by a Hargreaves assay (Fig. $5 \mathrm{H}$; $N=7, p=0.19)$. These results support our prediction that Tuba1 $\mathrm{N}^{\mathrm{ND/+}}$-induced behavioral deficits are not restricted to the motor system, but rather appear to preferentially impact the far-reaching neurons that innervate the hindlimbs. The degenerative behavioral phenotypes in Tuba1a ${ }^{N D /+}$ mice indicate that Tuba1a deficiency has long-lasting consequences for neuronal function.

\section{TUBA1A deficit impairs hindlimb behavior without apparent neuronal cell death or axon degeneration}

Complex motor behaviors involve multiple types of motor neurons coordinated through multiple brain regions including the cortex, cerebellum, and spinal cord. We examined survival of neurons that are important for coordinated movement in each of these systems in Tuba1 $a^{N D /+}$ and wild-type mice. Cortical lamination occurs normally in Tuba1 $1 \mathrm{~N}^{\mathrm{ND} / \mathrm{+}}$ mice, and there was no evidence of heterotopic neurons in Tuba1 $a^{N D /+}$ brains (Fig. $6 A ; N=3$ ). To assess cortical motor neuron number, we used the transcription factor ER81 as a marker of Layer V cortical neurons that project to the spinal cord (Yoneshima et al., 2006). We found no difference in the number of cortical motor neurons in Layer $\mathrm{V}$ of the motor cortex and no evidence of apoptosis before or after the onset of quantifiable motor deficits in Tuba1 $\mathrm{N}^{N /+}$ and wild type (Fig. 6A,B; Extended Data Fig. $6-1 ; p=0.1$ ). We quantified the number of Purkinje neurons, the primary output cells of the cerebellum, and found no difference in Purkinje cell number between genotypes at either time point (Fig. $6 A, C ; N=3$, $p=0.12)$. Quantification of motor neurons in serial sections through the lumbar spinal cord, which contains neurons that innervate the rear limbs, revealed that there was no alteration to the number of spinal motor neurons in Tuba1a $\mathrm{ND}^{\mathrm{N} /+}$ animals compared with wild type (Fig. 6A,D; 
A
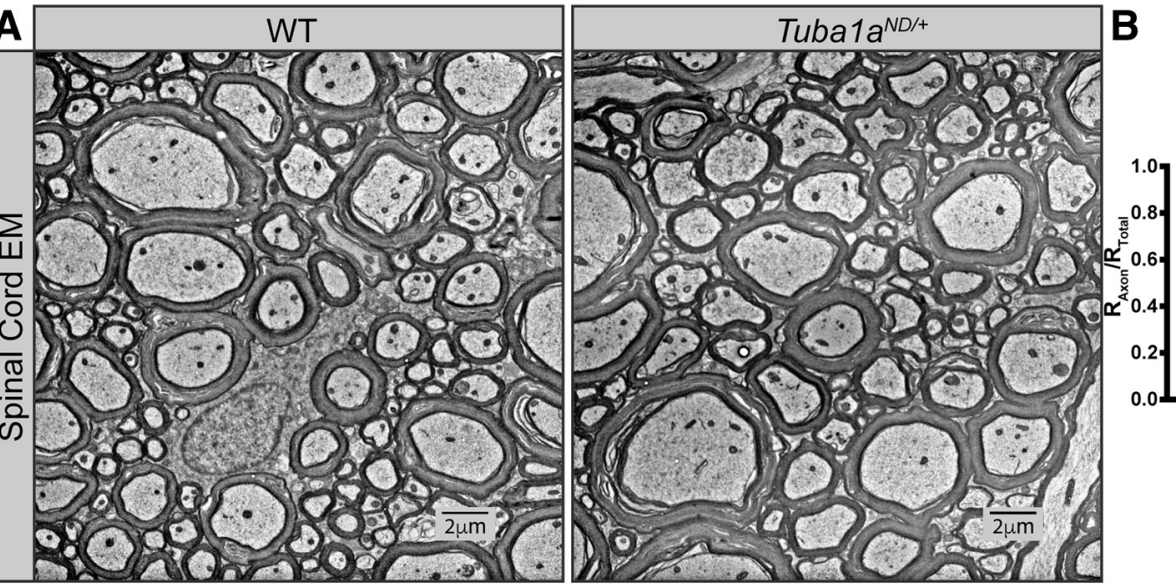

C

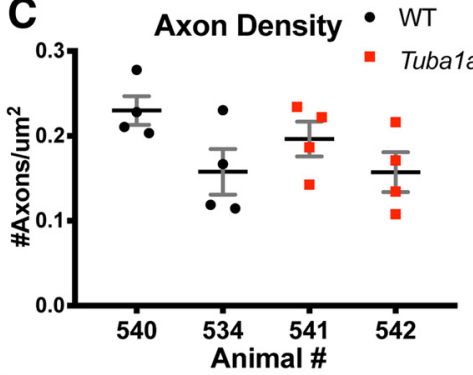

Axon Diameter
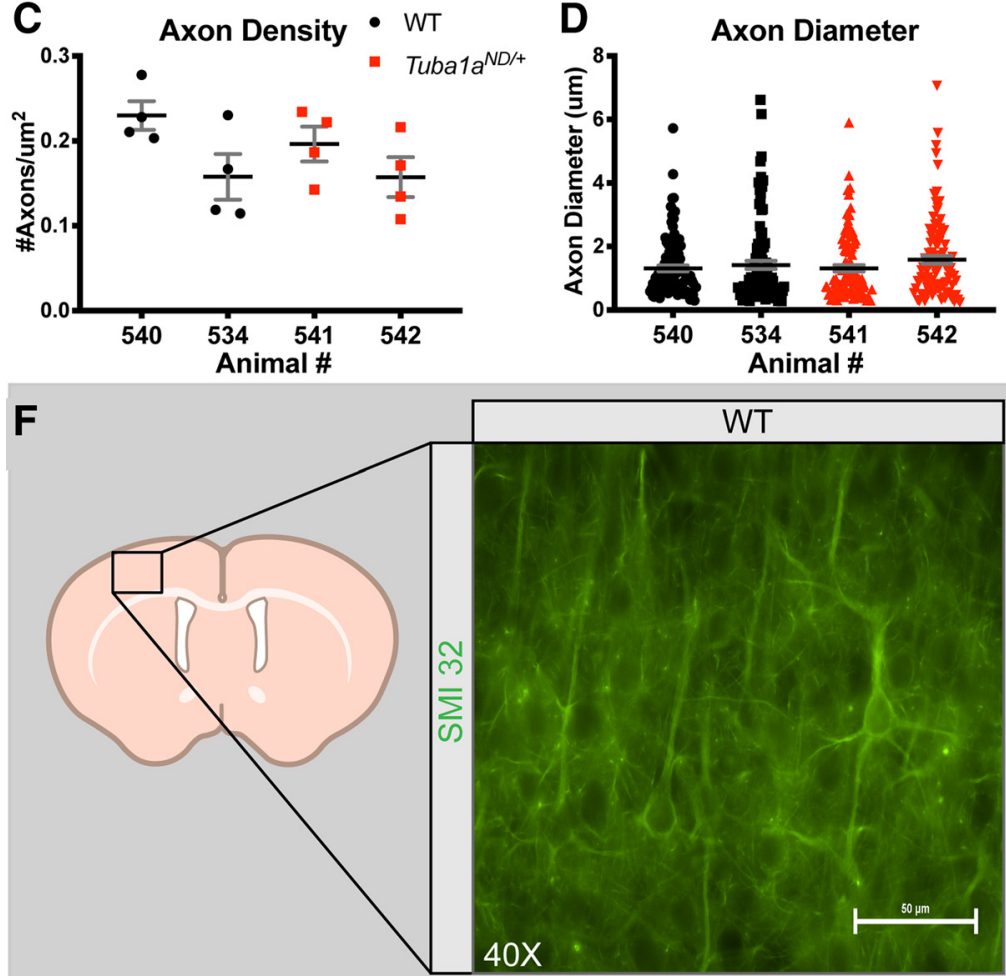

G

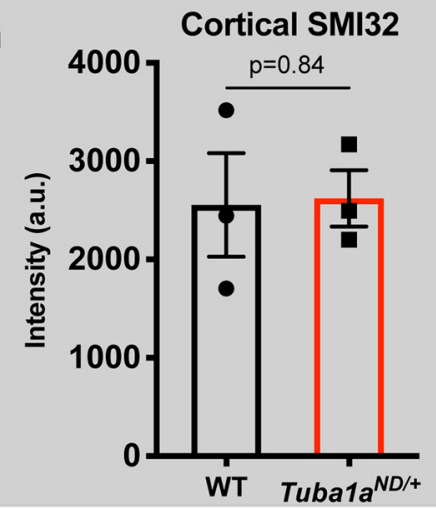

H
E

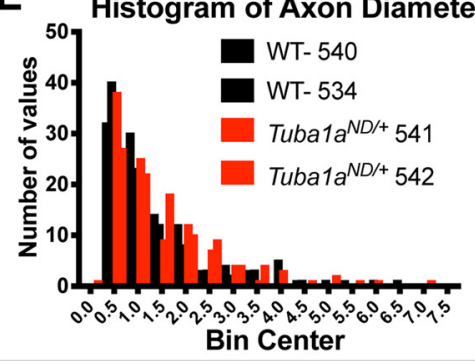

G-ratio • WT

- Tuba1a $a^{N D /+}$

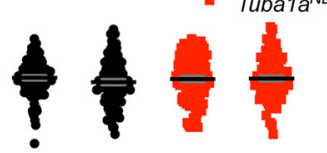

$\begin{array}{llll}540 & 534 & 541 & 542\end{array}$

Animal \#
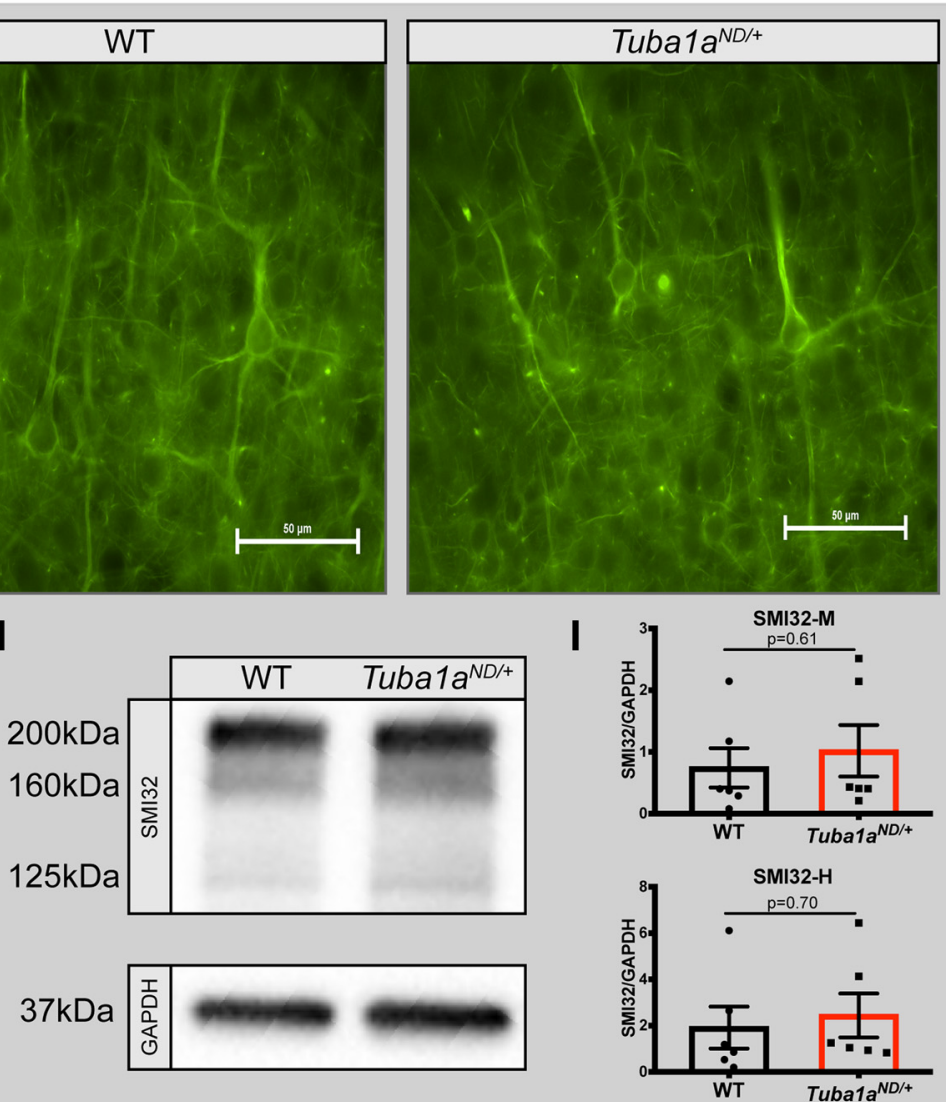

Figure 7. No deficits in axon morphology, myelination, or survival in Tuba1a ${ }^{N D /+}$ mice. $\boldsymbol{A}$, Electron micrographs of spinal cord axons in cross-section for adult wild-type (left) and Tuba $1 a^{N D /+}$ (right) mice. Six images per animal and two animals per genotype were assessed for EM analyses. B, Scatter plot displaying myelin thickness measure (G-ratio) for two wild-type and two Tuba1a ${ }^{N D /+}$ mice $(N=2$ mice, $n=100$ axons, $p=0.42)$. C , Scatter plot of axon density quantified in electron micrograph images for adult wild-type and Tuba1a ${ }^{N D /+}$ mice $(N=2$ mice, $n=6$ fields, $p=0.72)$. $\boldsymbol{D}$, Scatter plot of axon diameter quantified in electron micrograph images 


\section{continued}

for adult wild-type and Tuba1 $a^{N D /+}$ mice $(N=2$ mice, $n=100$ axons, $p=0.63)$. $E$, Histogram representing the distribution of axon diameters from data in $\boldsymbol{D}, \boldsymbol{F}$, Anti-SMI-32 (non-phosphorylated neurofilament) immunofluorescence images in cortex of adult wildtype (left) and Tuba1a ${ }^{N D /+}$ mice (right); $40 \times$ magnification. Scale bar: $10 \mu \mathrm{m}$. G, Bar graph representing SMI-32 intensity in a cortical region of interest for wild-type and Tuba1 $\mathrm{a}^{\mathrm{ND/+}}$ mice. Data points represent regions of interest, with three animals per genotype assessed $(p=0.77)$. $\boldsymbol{H}$, Western blotting for SMI-32 in whole-brain lysates from adult wild-type and Tuba1a ${ }^{\mathrm{ND} /+}$ mice. Bands for heavy (200 kDa), medium (160 kDa), and light (125 kDa) chain non-phosphorylated neurofilament shown (top). GAPDH used for normalization (37 kDa; bottom). Data points represent technical replicates with three biological replicates performed. I, Bar graphs representing quantification of SMI-32 medium (right, $p=0.61$ ) and heavy (left, $p=0.70$ ) expression in whole-brain lysates, normalized to GAPDH. For all graphs, bar or line indicates mean and error bars display SEM.

$N=3, p=0.2)$. In electron micrographs of mature spinal cords from Tuba1a $\mathrm{ND/}^{\mathrm{N}}$ and wild-type animals (Fig. $7 A$; $N=2$ mice), we found no difference in myelin thickness by G-ratio (Fig. 7B; $n=100$ axons, $p=0.42$ ), axon density (Fig. $7 C ; p=0.72$ ), or axon diameter (Fig. $7 D, E ; p=0.63$ ) at three or 10 months of age. Reduced neuron survival, loss of myelination, and axon degeneration are reported in several movement disorders (De Vos et al., 2008; Murray et al., 2010; Beharry et al., 2014; Brady and Morfini, 2017; Correale et al., 2019; Andreone et al., 2020). However, these results indicate that reduced TUBA1A function does not impact the establishment or maintenance of spinal motor neuron density, axon caliber, or myelination.

We examined the possibility that reduced TUBA1A function causes axon degeneration. To evaluate axon integrity in the Tuba1 $a^{N D /+}$ model, we quantified non-phosphorylated neurofilament (SMI32) that is highly abundant in degenerating axons (Watson et al., 1993; Thangavel et al., 2009; Seehusen and Baumgärtner, 2010). We sampled several regions of interest from cortex and observed no increase in SMI32 immunoreactivity in cortical sections from Tuba1 $a^{N D /+}$ mice compared with wild type (Fig. 7F,G; $N=4, p=0.84$ ). We also quantified SMI32 abundance in Tuba $1 \mathrm{a}^{\mathrm{ND/+}}$ and wild-type whole-brain lysates and detected no difference in SMI32 protein between the two groups (Fig. $7 H, l ; N=3$, NFT-M $p=0.61$, NFT-H $p=0.70)$. Overall, these data support the conclusion that reduction of TUBA1A causes adult-onset behavioral deficits without obvious detriment to neuronal survival or morphology.

\section{NMJ synapses are diminished by prolonged microtubule dysfunction}

Finally, we examined whether Tuba1 $a^{N D /+}$ impacts synaptic structure at the NMJ. NMJ function is critical to motor output, as it allows for the conversion of electrochemical signaling in neurons to muscle contraction and movement. To assess NMJ synaptic morphology, we labeled acetylcholine receptors (AChRs) with fluorescently conjugated $\alpha$-bungarotoxin in EDL muscles of both juvenile and adult Tuba $1 a^{N D /+}$ and wild-type hindlimbs. In rodents, NMJ synapses begin forming embryonically, and complete the final stages of synapse maturation, including synaptic pruning and stabilization, between two and three weeks postnatal (Tintignac et al., 2015). Juvenile Tuba1a $a^{N D /+}$ NMJ synapses were evaluated at one month of age, before the onset of quantifiable behavioral deficits, but following the completion of most NMJ development. Wild-type and Tuba1a $a^{N D /+}$ synapses labeled for postsynaptic AChRs as well as presynaptic vesicle marker synaptophysin were evaluated for synaptic area and the relative density of both presynaptic and postsynaptic machinery (Fig. 8A). Juvenile Tuba1a ${ }^{N D /+}$ EDL NMJ synapses were indistinguishable from wild type in synaptic area (Fig. $8 B ; n=69$ synapses, $p=0.3$ ), density of presynaptic and postsynaptic components (Fig. $8 D, C ; N=3$ mice, $n=39$ synapses, $p>0.5$ for both), and the ratio of presynaptic to postsynaptic machinery (Fig. 8E; $n=39$ synapses, $p=0.74)$. The same analyses were performed for EDL synapses in adult (approximately one year old) wild-type and Tuba1a $a^{N D /+}$ mice. A dramatic reduction in synaptic branching complexity in the Tuba1aND/+ EDL was immediately apparent, illustrated by the example images (Fig. 8A). Further, in adult Tuba1 ${ }^{N D /+}$ NMJs, synaptic area was found to be significantly reduced compared with wild type (Fig. 8F; $N=3$ mice, $n=45$ synapses, $p<0.0001)$. Additionally, adult Tuba1a ${ }^{N D /+}$ synapses contained a higher density of postsynaptic AChRs than wild type (Fig. 8G; $p<0.0001$ ). The increased AChR density in the adult EDL was also accompanied by an increase in presynaptic synaptophysin $(8 \mathrm{H})$, but the ratio of presynaptic to postsynaptic machinery was modestly decreased in Tuba $1 a^{N D /+}$ NMJs compared with wild type, indicating that Tuba1a ${ }^{N D /+}$ NMJs had lower presynaptic vesicle density relative to the postsynaptic receptor density (Fig. $8 ; p<0.0001$ and $p=0.03$, respectively). Collectively these data demonstrate that reduced TUBA1A diminishes NMJ size and alters NMJ synapse morphology and functional properties in an age-related manner.

\section{Discussion}

Here, we show that significant reduction in developmental total $\alpha$-tubulin resulting from a loss of function mutation in Tuba1a (Tuba1a ${ }^{N D /+}$ ) causes an adult onset movement disorder. We show that assembly of the microtubule network is altered resulting in disrupted organelle trafficking early in development in cultured Tuba1a $\mathrm{ND}^{\mathrm{ND}+}$ cortical neurons, suggesting that neuronal microtubule networks are not set up correctly when there is not adequate $\alpha$-tubulin available (Figs. 1-4). While these aberrant neuronal microtubule networks are sufficient to establish juvenile NMJ synapses similarly to wild type, Tuba1 $a^{N D /+}$ NMJ synapses deteriorate over time (Fig. 8), potentially contributing to adult-onset motor deficits. This work provides the first evidence that the developmental tubulin TUBA1A is necessary for adult neuronal function and suggests that mature neurons require TUBA1A for proper function throughout their lifetime. 

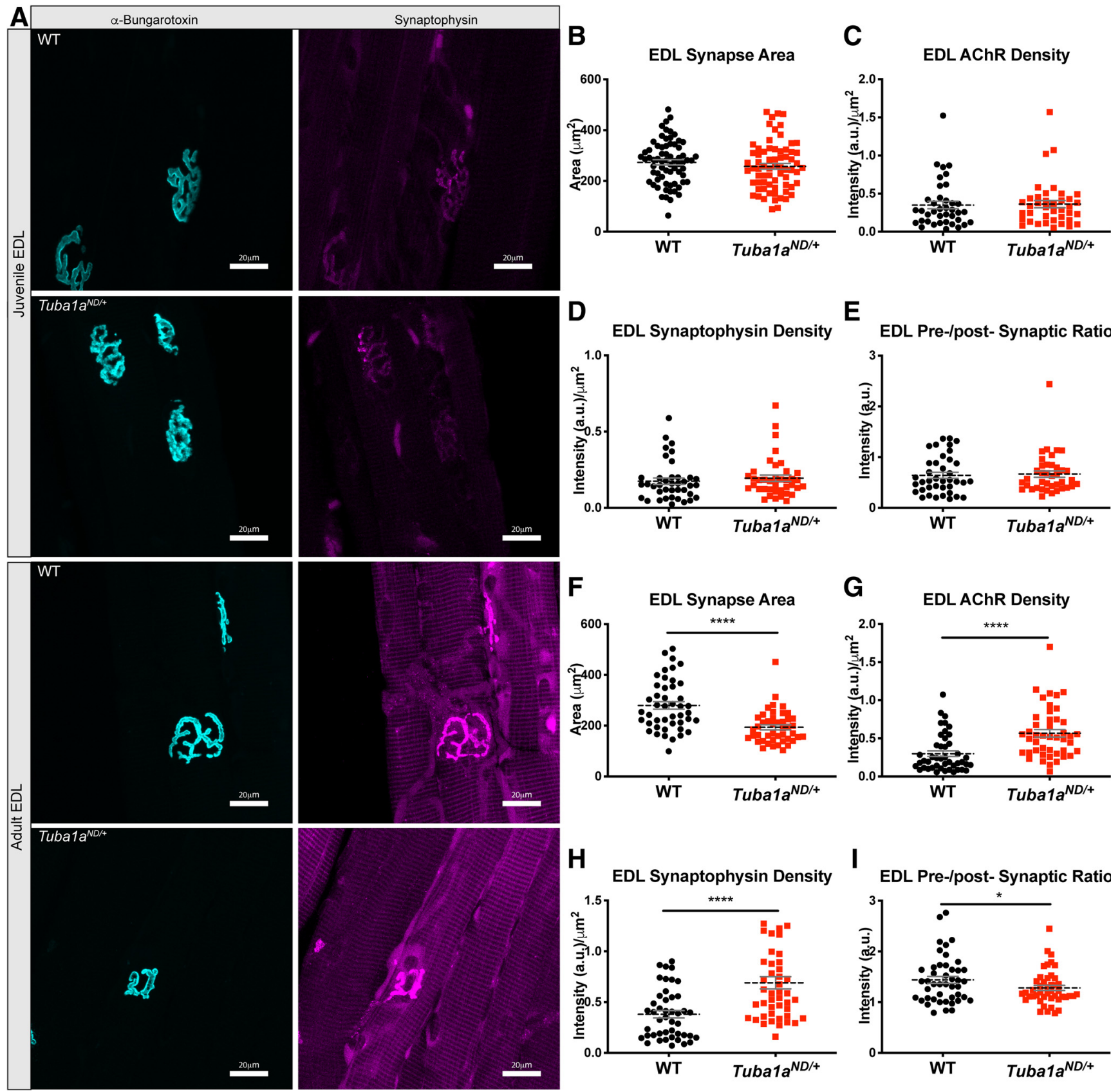

E
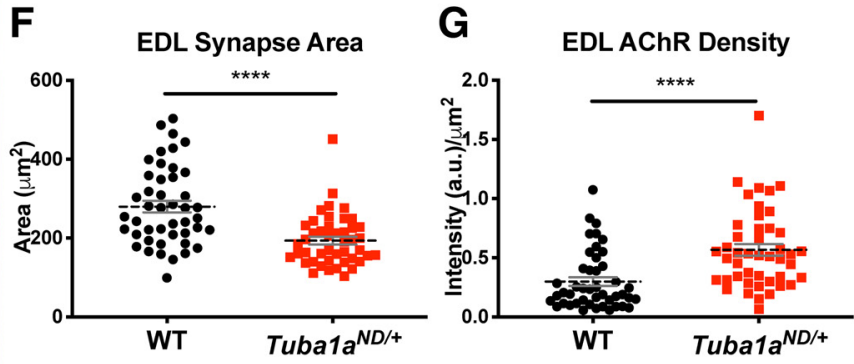

H

EDL Synaptophysin Density

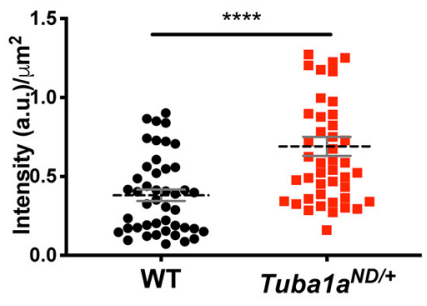

I

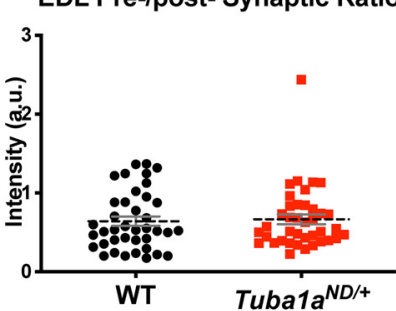

Figure 8. Neuromuscular Junction (NMJ) synapses deteriorate over time in Tuba1 $a^{N D /+}$ mice. $\boldsymbol{A}$, Teased Extensor digitorum longus (EDL) muscle fibers labeled with $\alpha$-bungarotoxin (cyan) and synaptophysin (magenta) for one-month-old (top) and one-year-old (bottom) wild-type and Tuba $1 a^{N D /+}$ mice. Muscle fibers from three mice per genotype at each time point were assessed. $\boldsymbol{B}$, Scatter plot of EDL synaptic $\left(\mu \mathrm{m}^{2}\right)$ measured from $\alpha$-bungarotoxin labeling in juvenile wild-type and Tuba1a ${ }^{N D /+}$ mice $(N=3$ mice, $n=69$ synapses, $p=0.43$ ). C , Scatter plot of postsynaptic acetylcholine receptors (AChRs) density measured as synaptic $\alpha$-bungarotoxin intensity in arbitrary units (a.u.) divided by synaptic area in juvenile mice ( $N=3$ mice, $n=39$ synapses, $p=0.89)$. $\boldsymbol{D}$, Scatter plot of presynaptic vesicle density measured as synaptic synaptophysin intensity divided by synaptic area in juvenile mice $(N=3$ mice, $n=39$ synapses, $p=0.54$ ). $\boldsymbol{E}$, Scatter plot of the ratio of synaptophysin to AChR (presynaptic to postsynaptic) intensity in juvenile wild-type and Tuba1a $a^{N D /+}$ mice $(N=3$ mice, $n=39$ synapses, $p=0.74)$. $\boldsymbol{F}$, Scatter plot of EDL synaptic area measured from $\alpha$-bungarotoxin labeling in adult wild-type and Tuba1 $a^{N D /+}$ mice $(N=3$ mice, $n=45$ synapses, $p<0.0001)$. G, Scatter plot of postsynaptic AChR density measured as synaptic $\alpha$-bungarotoxin intensity divided by synaptic area in adult mice $(N=3$ mice, $n=45$ synapses, $p<0.0001)$. $\boldsymbol{H}$, Scatter plot of presynaptic vesicle density measured as synaptic synaptophysin intensity divided by synaptic area in adult mice $(N=3, n=45, p<0.0001)$. I, Scatter plot of the ratio of synaptophysin to AChR (presynaptic to postsynaptic) intensity in adult wild-type and Tuba1 $a^{N D /+}$ mice $(N=3$ mice, $n=45$ synapses, $p=0.03)$. For all graphs, data points represent individual synapses with line representing mean of data \pm SEM. Data were nested by animal and analyzed for statistical significance between genotypes by a two-way ANOVA. $* p<0.05 ; * * * * p<0.0001$. 
Table 1: Statistical table

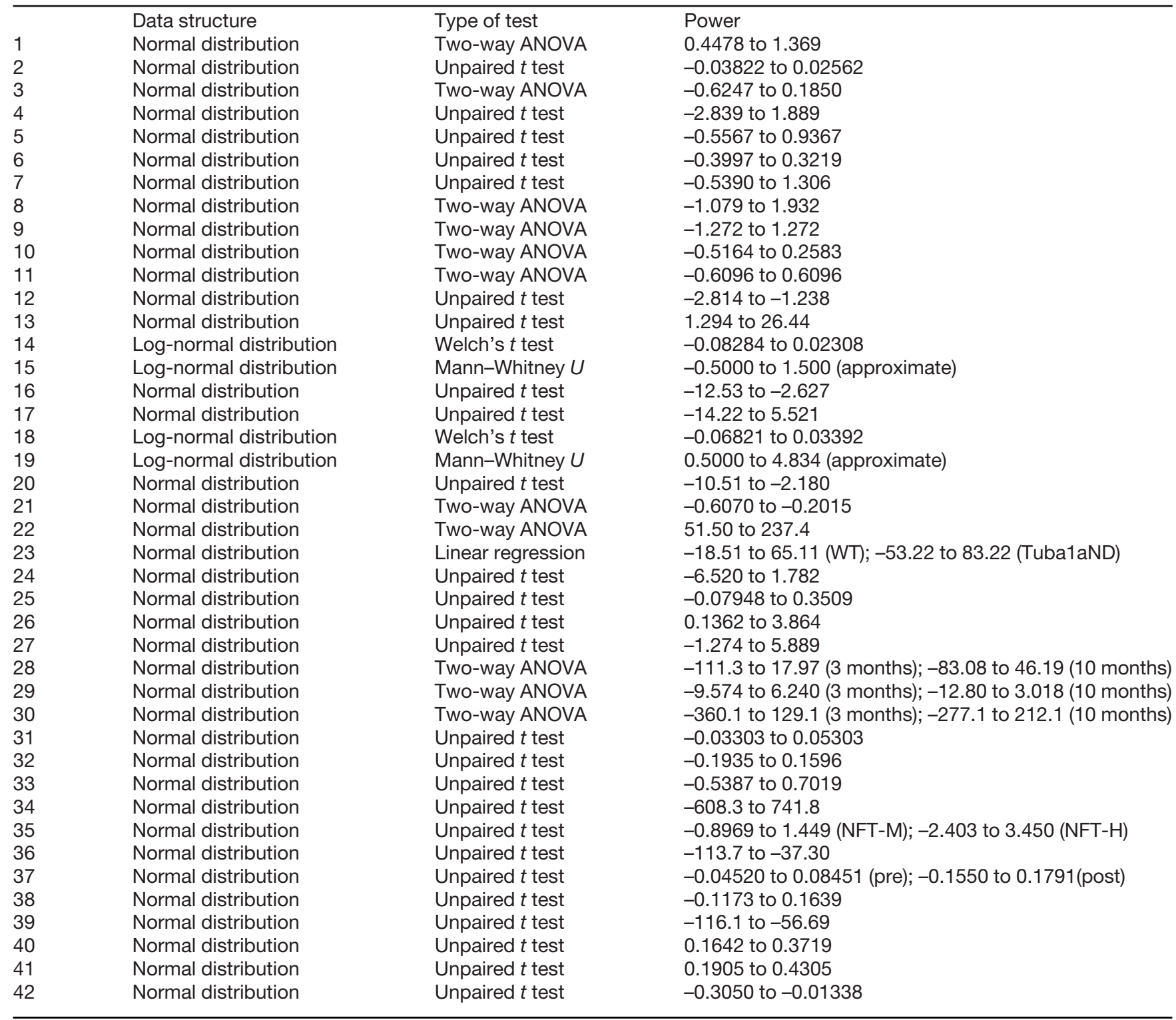

The microtubule cytoskeleton forms the tracks on which motor proteins bind to facilitate intracellular transport. Mitochondria and lysosomes are both transported by dynein motor proteins in the retrograde direction, but differ in the kinesin motors that primarily regulate their anterograde movement, with lysosome transport dominated by kinesin2 and mitochondrial transport occurring primarily by kinesin-1 (Tanaka et al., 1998; Ligon and Steward, 2000; Hendricks et al., 2010). Thus, examining transport dynamics for these two different organelles allowed us to distinguish between compromised interactions with specific motor proteins or universal effects of a compromised microtubule network. Neither lysosome nor mitochondria velocity was affected by depletion of TUBA1A function, suggesting that motors interact with the microtubule substrate normally (Fig. 4D,H). Importantly, we did not see differences in amounts of PTMs that are known to affect the activity of motors on their microtubule substrates (Fig. 2C,
$D)$. Thus, this is the first demonstration that reducing available TUBA1A affects organelle trafficking in living neurons.

Mitochondria and lysosomes move with similar average velocities in wild-type neurons, however the dynamics of movement for these two organelle types vary greatly. We observed a larger proportion of stationary mitochondria $(60 \%)$ than stationary lysosomes $(30 \%)$ in wild-type neurons (Fig. $4 C, G$ ). The proportion of stationary mitochondria in our cortical cultures was consistent with what has been previously reported in neurons (Ligon and Steward, 2000; Misgeld et al., 2007; Schwarz, 2013). The variation in transport dynamics between these two organelle types could potentially explain the discrepancy in trafficking deficits we observed between lysosomes and mitochondria in Tuba1a ${ }^{N D /+}$ neurons. Lysosomes are highly motile organelles; therefore, a lysosomal stalling deficit was readily detected in Tuba1 $a^{N D /+}$ neurons (Fig. 4C). However, such a deficit was not apparent in the relatively stationary 
mitochondrial population (Fig. 4G). The relatively subtle deficit in pause duration that was observed in mitochondria was not detectable in lysosomes, which had much shorter pause durations on average (Fig. 4E,I). Despite the discrepancies in how these abnormalities were detected between different organelles, the overall distance traveled by mitochondria and lysosomes was consistently reduced in Tuba1a ${ }^{N D /+}$ neurons compared with wild type (Fig. 4F,J). The increase in pausing or stalling behavior (Fig. $4 C, l$ ), combined with the reduced $\alpha$-tubulin protein during development, and normal neuronal survival (Figs. 2, 6, 7) suggests that reduced availability of TUBA1A is detrimental to adult neuronal function, but not neuronal survival.

The abundance of $\alpha$-tubulin protein during brain development is reduced in Tuba1 $a^{N D /+}$ brains (Fig. 2). This deficit in $\alpha$-tubulin reduced the number of growing microtubule $(+)$-ends per micrometer of neurite in developing Tuba1a $\mathrm{ND}^{\mathrm{N}+}$ neurons, indicating the neuronal microtubule network contains a lower density of microtubules in Tuba1a ${ }^{N D /+}$ mice (Fig. 3). We did not observe differences in neuronal survival, axon caliber, myelination, or markers for axon degeneration in Tuba1 $a^{N D /+}$ compared with wildtype siblings (Figs. 6,7 ) suggesting that reduced TUBA1A impacts neuron function rather than neuron survival or axon morphology. Collectively, these results could suggest that adult Tuba1a expression may be important for maintenance of microtubule tracks in neurons and mutant TUBA1A $A^{N D}$ microtubules are not sufficient for that requirement. We favor a second model, that the developmental TUBA1A deficit does not allow a neuron to correctly establish microtubule networks of the appropriate density, and these defects cannot be sufficiently repaired to maintain functional NMJ synapses in adulthood despite adequate levels of adult $\alpha$-tubulin protein (Figs. 2, $3,8)$.

Intracellular transport deficits in Tuba1a ${ }^{N D /+}$ neurons provide a putative mechanism by which reduced TUBA1A impairs neuronal function to cause adult-onset behavioral deficits. However, as cortical neurons are not representative of the adult condition, follow-up studies are needed to determine whether trafficking deficits persist into adulthood in the Tuba1a ${ }^{N D /+}$ model. Because developmental $\alpha$-tubulin protein is reduced in Tuba $1 a^{N D /+}$ brains without detectable compensation from any other $\alpha$-tubulin isotypes (Fig. 2), we propose a model in which early $\alpha$-tubulin deficits cause formation of inadequate neuronal microtubule tracks that cannot be rescued by subsequent restoration of $\alpha$-tubulin protein. Over time, the consequences of early tubulin deficiency could become more pronounced and result in impairment of synaptic function and animal behavior. Microtubules in neurons are not singly nucleated from the centrosome, but rather exist in short segments that tile the length of the axons and dendrites (Stiess et al., 2010). When moving cargoes reach the end of a microtubule segment, the motor protein pauses to switch tracks (Yogev et al., 2016). Thus, the observed increase in organelle pausing behavior paired with developmental decreases in $\alpha$-tubulin protein and microtubule density could indicate that motor proteins in Tuba1 $a^{N D /+}$ neurons encounter microtubule ends more frequently, due to the presence of potential gaps in the tiled microtubule network. Alternatively, while no compensation for loss of TUBA1A was observed at the mRNA level, decreased $\alpha$-tubulin protein would likely shift the $\alpha$-tubulin isotype blend at the protein level. It has been previously shown that incorporation of different tubulin isotypes alters microtubule properties, whether directly or through effects on MAP binding (Panda et al., 1994; Sirajuddin et al., 2014; Gadadhar et al., 2017; Honda et al., 2017). Although $\alpha$-tubulin protein levels are similar to wild type in the adult Tuba1a $a^{N D /+}$ brain, it is unclear whether adult Tuba1 $a^{N D /+}$ microtubule polymers contain normal $\alpha$-tubulin isotype compositions.

Microtubules and intracellular transport are important for synaptic signaling and maintenance (Kim and Lisman, 2001; Jaworski et al., 2009; Maas et al., 2009; Stephan et al., 2015). Neurons are morphologically complex cells, with processes often extending long distances from the soma, and are thus highly dependent on functional intracellular transport. Transport deficits, such as those observed in the Tuba1a ${ }^{N D /+}$ model could impair both delivery and clearance of cargoes if persistent over time, leading to neuronal dysfunction. Functional intracellular transport is essential for delivery of mitochondria and synaptic components to the presynapse (Goldstein et al., 2008). Thus, the transport deficits caused by Tuba1a $a^{N D /+}$ substitution could alter synaptic transmission and thereby cause deterioration of synapse morphology, potentially explaining the observed NMJ deficits (Fig. 8). The increased density of both presynaptic and postsynaptic machinery that was observed in the Tuba1a ${ }^{N D /+} \mathrm{NMJ}$ (Fig. $8 G, H$ ) suggests a possible impairment in clearance of vesicles from the presynapse and receptors from the postsynapse. Moreover, the reduction in NMJ synaptic area (Fig. $8 F$ ) and branching complexity indicates that the increased density of AChRs and presynaptic vesicles may be a form of compensation for improperly functioning synapses. This hypothesis is further supported by the observed dysregulation of presynaptic to postsynaptic component ratios in adult Tuba1a ${ }^{N D /+}$ synapses (Fig. 8/). NMJ synapses are generally quite stable over time, but are known to undergo morphologic changes in response to processes that alter synaptic transmission, such as diseases of the neuromuscular system and aging (Murray et al., 2008, 2010; Jones et al., 2017; Liu et al., 2017). Decreased NMJ size explains impaired motor behaviors in Tuba1a ${ }^{N /+}$ mice (Fig. $5 A-C$ ). However, given that Tuba1a is expressed in all neurons, and Tuba $1 a^{N D /+}$ mice also exhibited degenerative sensory deficits (Fig. 5G,H), we do not expect that the NMJ is the only synapse type affected by deficiency in TUBA1A, but this idea remains to be tested. The prominence of hindlimb-specific behavioral phenotypes in the Tuba1a ${ }^{N D /+}$ mouse model suggests that axon length may predict sensitivity to tubulin deficiency.

Microtubule dysfunction, including intracellular trafficking defects, have been described in nearly every neurodegenerative disease of the nervous system, and can precede other signs of neuronal dysfunction (Reynolds et al., 2004; De Vos et al., 2008; Cartelli et al., 2010; 
Matamoros and Baas, 2016; Brady and Morfini, 2017). Although there is abundant evidence of microtubule dysfunction in neurodegenerative disease, it has been difficult to pinpoint how or whether the microtubule cytoskeleton contributes to disease etiology. Microtubule dynamics, PTMs, and MAP interactions become dysregulated in neurodegenerative diseases such as Alzheimer's and Parkinson's disease, and amyotrophic lateral sclerosis (ALS); however, it has remained unclear as to whether cytoskeletal dysfunction is causative of disease or merely correlated with its progression (Cartelli et al., 2010; Rogowski et al., 2010; Brunden et al., 2011; Dubey et al., 2015; Matamoros and Baas, 2016). Mutations that disrupt the neuronal $\alpha$-tubulin TUBA4A have been identified familial and sporadic ALS (Rademakers and van Blitterswijk, 2014; Smith et al., 2014; Pensato et al., 2015; Li et al., 2018). The mechanisms by which TUBA4A mutations may cause neurodegeneration remain unclear. Using the Tuba $1 a^{N D /+}$ model, we have demonstrated that loss-offunction in a major neuronal $\alpha$-tubulin is sufficient to induce adult-onset behavioral degeneration. Further, we have shown that Tuba1 $1 a^{N D /+}$ does not induce neuronal death or degeneration, but rather causes synaptic abnormalities, potentially by impairing intracellular transport. We provide direct evidence that deficits in microtubule function can cause degenerative neuronal pathology at the molecular, cellular, and behavioral level.

\section{References}

Aiken J, Buscaglia G, Bates EA, Moore JK (2017) The $\alpha$-tubulin gene TUBA1A in brain development: a key ingredient in the neuronal isotype blend. J Dev Biol 5:8.

Aiken J, Buscaglia G, Aiken AS, Moore JK, Bates EA (2019a) Tubulin mutations in brain development disorders: why haploinsufficiency does not explain TUBA1A tubulinopathies. Cytoskeleton (Hoboken). Advance online publication. Retrieved October 1, 2019.

Aiken J, Moore JK, Bates EA (2019b) TUBA1A mutations identified in lissencephaly patients dominantly disrupt neuronal migration and impair dynein activity. Hum Mol Genet 28:1227-1243.

Andreone BJ, Larhammar M, Lewcock JW (2020) Cell death and neurodegeneration. Cold Spring Harb Perspect Biol 12:a036434.

Audebert S, Koulakoff A, Berwald-Netter Y, Gros F, Denoulet P, Edde B (1994) Developmental regulation of polyglutamylated alpha- and beta-tubulin in mouse brain neurons. J Cell Sci 107:2313-2322.

Bamji SX, Miller FD (1996) Comparison of the expression of a T alpha 1:nlacZ transgene and T alpha 1 alpha-tubulin mRNA in the mature central nervous system. J Comp Neurol 374:52-69.

Beharry C, Cohen LS, Di J, Ibrahim K, Briffa-Mirabella S, Alonso Adel C (2014) Tau-induced neurodegeneration: mechanisms and targets. Neurosci Bull 30:346-358.

Bittermann E, Abdelhamed Z, Liegel RP, Menke C, Timms A, Beier DR, Stottmann RW (2019) Differential requirements of tubulin genes in mammalian forebrain development. PLoS Genet 15: e1008243.

Bonin RP, Bories C, De Koninck Y (2014) A simplified up-down method (SUDO) for measuring mechanical nociception in rodents using von Frey filaments. Mol Pain 10:26.

Bonnet C, Boucher D, Lazereg S, Pedrotti B, Islam K, Denoulet P, Larcher JC (2001) Differential binding regulation of microtubuleassociated proteins MAP1A, MAP1B, and MAP2 by tubulin polyglutamylation. J Biol Chem 276:12839-12848.
Brady ST, Morfini GA (2017) Regulation of motor proteins, axonal transport deficits and adult-onset neurodegenerative diseases. Neurobiol Dis 105:273-282.

Brady ST, Tytell M, Lasek RJ (1984) Axonal tubulin and axonal microtubules: biochemical evidence for cold stability. J Cell Biol 99:1716-1724.

Brunden KR, Yao Y, Potuzak JS, Ferrer NI, Ballatore C, James MJ, Hogan AM, Trojanowski JQ, Smith AB 3rd, Lee VM (2011) The characterization of microtubule-stabilizing drugs as possible therapeutic agents for Alzheimer's disease and related tauopathies. Pharmacol Res 63:341-351.

Cartelli D, Ronchi C, Maggioni MG, Rodighiero S, Giavini E, Cappelletti G (2010) Microtubule dysfunction precedes transport impairment and mitochondria damage in MPP+ -induced neurodegeneration. J Neurochem 115:247-258.

Correale J, Marrodan M, Ysrraelit MC (2019) Mechanisms of neurodegeneration and axonal dysfunction in progressive multiple sclerosis. Biomedicines 7:14.

Cushion TD, Dobyns WB, Mullins JG, Stoodley N, Chung SK, Fry AE, Hehr U, Gunny R, Aylsworth AS, Prabhakar P, Uyanik G, Rankin J, Rees MI, Pilz DT (2013) Overlapping cortical malformations and mutations in TUBB2B and TUBA1A. Brain 136:536-548.

De Vos KJ, Grierson AJ, Ackerley S, Miller CC (2008) Role of axonal transport in neurodegenerative diseases. Annu Rev Neurosci 31:151-173.

Dubey J, Ratnakaran N, Koushika SP (2015) Neurodegeneration and microtubule dynamics: death by a thousand cuts. Front Cell Neurosci 9:343.

Fallet-Bianco C, Loeuillet L, Poirier K, Loget P, Chapon F, Pasquier L, Saillour Y, Beldjord C, Chelly J, Francis F (2008) Neuropathological phenotype of a distinct form of lissencephaly associated with mutations in TUBA1A. Brain 131:2304-2320.

Gadadhar S, Bodakuntla S, Natarajan K, Janke C (2017) The tubulin code at a glance. J Cell Sci 130:1347-1353.

Gartz Hanson M, Aiken J, Sietsema DV, Sept D, Bates EA, Niswander L, Moore JK (2016) Novel $\alpha$-tubulin mutation disrupts neural development and tubulin proteostasis. Dev Biol 409:406419.

Gloster A, Wu W, Speelman A, Weiss S, Causing C, Pozniak C, Reynolds B, Chang E, Toma JG, Miller FD (1994) The T alpha 1 alpha-tubulin promoter specifies gene expression as a function of neuronal growth and regeneration in transgenic mice. J Neurosci 14:7319-7330.

Gloster A, El-Bizri H, Bamji SX, Rogers D, Miller FD (1999) Early induction of Talpha1 alpha-tubulin transcription in neurons of the developing nervous system. J Comp Neurol 405:45-60.

Goldstein AY, Wang X, Schwarz TL (2008) Axonal transport and the delivery of pre-synaptic components. Curr Opin Neurobiol 18:495503.

Hendricks AG, Perlson E, Ross JL, Schroeder HW 3rd, Tokito M, Holzbaur EL (2010) Motor coordination via a tug-of-war mechanism drives bidirectional vesicle transport. Curr Biol 20:697-702.

Heo S, Diering GH, Na CH, Nirujogi RS, Bachman JL, Pandey A, Huganir RL (2018) Identification of long-lived synaptic proteins by proteomic analysis of synaptosome protein turnover. Proc Natl Acad Sci USA 115:E3827-E3836.

Honda Y, Tsuchiya K, Sumiyoshi E, Haruta N, Sugimoto A (2017) Tubulin isotype substitution revealed that isotype combination modulates microtubule dynamics in C. elegans embryos. J Cell Sci 130:1652-1661.

Hurd DD, Saxton WM (1996) Kinesin mutations cause motor neuron disease phenotypes by disrupting fast axonal transport in Drosophila. Genetics 144:1075-1085.

Jaworski J, Kapitein LC, Gouveia SM, Dortland BR, Wulf PS, Grigoriev I, Camera P, Spangler SA, Di Stefano P, Demmers J, Krugers H, Defilippi P, Akhmanova A, Hoogenraad CC (2009) Dynamic microtubules regulate dendritic spine morphology and synaptic plasticity. Neuron 61:85-100.

Jones RA, Harrison C, Eaton SL, Llavero Hurtado M, Graham LC, Alkhammash L, Oladiran OA, Gale A, Lamont DJ, Simpson H, 
Simmen MW, Soeller C, Wishart TM, Gillingwater TH (2017) Cellular and molecular anatomy of the human neuromuscular junction. Cell Rep 21:2348-2356.

Keays DA, Tian G, Poirier K, Huang GJ, Siebold C, Cleak J, Oliver PL, Fray M, Harvey RJ, Molnár Z, Piñon MC, Dear N, Valdar W, Brown SD, Davies KE, Rawlins JN, Cowan NJ, Nolan P, Chelly J, Flint J (2007) Mutations in alpha-tubulin cause abnormal neuronal migration in mice and lissencephaly in humans. Cell 128:45-57.

Kim CH, Lisman JE (2001) A labile component of AMPA receptormediated synaptic transmission is dependent on microtubule motors, actin, and $\mathrm{N}$-ethylmaleimide-sensitive factor. J Neurosci 21:4188-4194.

Kumar RA, Pilz DT, Babatz TD, Cushion TD, Harvey K, Topf M, Yates L, Robb S, Uyanik G, Mancini GM, Rees MI, Harvey RJ, Dobyns WB (2010) TUBA1A mutations cause wide spectrum lissencephaly (smooth brain) and suggest that multiple neuronal migration pathways converge on alpha tubulins. Hum Mol Genet 19:2817-2827.

Lewis SA, Lee MG, Cowan NJ (1985) Five mouse tubulin isotypes and their regulated expression during development. $\mathrm{J}$ Cell Biol 101:852-861.

Li J, He J, Tang L, Chen L, Ma Y, Fan D (2018) Screening for TUBA4A mutations in a large Chinese cohort of patients with ALS: re-evaluating the pathogenesis of TUBA4A in ALS. J Neurol Neurosurg Psychiatry 89:1350-1352.

Ligon LA, Steward O (2000) Movement of mitochondria in the axons and dendrites of cultured hippocampal neurons. J Comp Neurol 427:340-350.

Liu W, Klose A, Forman S, Paris ND, Wei-LaPierre L, Cortés-Lopéz M, Tan A, Flaherty M, Miura P, Dirksen RT, Chakkalakal JV (2017) Loss of adult skeletal muscle stem cells drives age-related neuromuscular junction degeneration. Elife 6:e26464.

Maas C, Belgardt D, Lee HK, Heisler FF, Lappe-Siefke C, Magiera MM, van Dijk J, Hausrat TJ, Janke C, Kneussel M (2009) Synaptic activation modifies microtubules underlying transport of postsynaptic cargo. Proc Natl Acad Sci USA 106:8731-8736.

Maday S, Twelvetrees AE, Moughamian AJ, Holzbaur EL (2014) Axonal transport: cargo-specific mechanisms of motility and regulation. Neuron 84:292-309.

Matamoros AJ, Baas PW (2016) Microtubules in health and degenerative disease of the nervous system. Brain Res Bull 126:217-225.

Miller FD, Naus CC, Durand M, Bloom FE, Milner RJ (1987) Isotypes of alpha-tubulin are differentially regulated during neuronal maturation. J Cell Biol 105:3065-3073.

Misgeld T, Kerschensteiner M, Bareyre FM, Burgess RW, Lichtman JW (2007) Imaging axonal transport of mitochondria in vivo. Nat Methods 4:559-561.

Murray LM, Comley LH, Thomson D, Parkinson N, Talbot K, Gillingwater TH (2008) Selective vulnerability of motor neurons and dissociation of pre- and post-synaptic pathology at the neuromuscular junction in mouse models of spinal muscular atrophy. Hum Mol Genet 17:949-962.

Murray LM, Talbot K, Gillingwater TH (2010) Review: neuromuscular synaptic vulnerability in motor neurone disease: amyotrophic lateral sclerosis and spinal muscular atrophy. Neuropathol Appl Neurobiol 36:133-156.

Natarajan K, Gadadhar S, Souphron J, Magiera MM, Janke C (2017) Molecular interactions between tubulin tails and glutamylases reveal determinants of glutamylation patterns. EMBO Rep 18:10131026.

Oegema R, Cushion TD, Phelps IG, Chung SK, Dempsey JC, Collins S, Mullins JG, Dudding T, Gill H, Green AJ, Dobyns WB, Ishak GE, Rees MI, Doherty D (2015) Recognizable cerebellar dysplasia associated with mutations in multiple tubulin genes. Hum Mol Genet 24:5313-5325.

Paden CM, Zhou X, Watt JA, Burton R, Pickett J, Oblinger MM (1995) Distribution of growth-associated class I alpha-tubulin and class II beta-tubulin mRNAs in adult rat brain. J Comp Neurol 362:368-384.
Panda D, Miller HP, Banerjee A, Ludueña RF, Wilson L (1994) Microtubule dynamics in vitro are regulated by the tubulin isotype composition. Proc Natl Acad Sci USA 91:11358-11362.

Pensato V, Tiloca C, Corrado L, Bertolin C, Sardone V, Del Bo R, Calini D, Mandrioli J, Lauria G, Mazzini L, Querin G, Ceroni M, Cantello R, Corti S, Castellotti B, Soldà G, Duga S, Comi GP, Cereda C, Sorarù G, et al. (2015) TUBA4A gene analysis in sporadic amyotrophic lateral sclerosis: identification of novel mutations. J Neurol 262:1376-1378.

Rademakers R, van Blitterswijk M (2014) Excess of rare damaging TUBA4A variants suggests cytoskeletal defects in ALS. Neuron 84:241-243.

Reynolds IJ, Malaiyandi LM, Coash M, Rintoul GL (2004) Mitochondrial trafficking in neurons: a key variable in neurodegeneration? J Bioenerg Biomembr 36:283-286.

Rogowski K, van Dijk J, Magiera MM, Bosc C, Deloulme JC, Bosson A, Peris L, Gold ND, Lacroix B, Bosch Grau M, Bec N, Larroque C, Desagher S, Holzer M, Andrieux A, Moutin MJ, Janke C (2010) A family of protein-deglutamylating enzymes associated with neurodegeneration. Cell 143:564-578.

Schwarz TL (2013) Mitochondrial trafficking in neurons. Cold Spring Harb Perspect Biol 5:a011304.

Seehusen F, Baumgärtner W (2010) Axonal pathology and loss precede demyelination and accompany chronic lesions in a spontaneously occurring animal model of multiple sclerosis. Brain Pathol 20:551-559.

Sirajuddin M, Rice LM, Vale RD (2014) Regulation of microtubule motors by tubulin isotypes and post-translational modifications. Nat Cell Biol 16:335-344.

Smith BN, Ticozzi N, Fallini C, Gkazi AS, Topp S, Kenna KP, Scotter EL, Kost J, Keagle P, Miller JW, Calini D, Vance C, Danielson EW, Troakes C, Tiloca C, Al-Sarraj S, Lewis EA, King A, Colombrita C, Pensato V, et al. (2014) Exome-wide rare variant analysis identifies TUBA4A mutations associated with familial ALS. Neuron 84:324331.

Song Y, Kirkpatrick LL, Schilling AB, Helseth DL, Chabot N, Keillor JW, Johnson GV, Brady ST (2013) Transglutaminase and polyamination of tubulin: posttranslational modification for stabilizing axonal microtubules. Neuron 78:109-123.

Stephan R, Goellner B, Moreno E, Frank CA, Hugenschmidt T, Genoud C, Aberle H, Pielage J (2015) Hierarchical microtubule organization controls axon caliber and transport and determines synaptic structure and stability. Dev Cell 33:5-21.

Stiess M, Maghelli N, Kapitein LC, Gomis-Rüth S, Wilsch-Bräuninger M, Hoogenraad CC, Tolić-Nørrelykke IM, Bradke F (2010) Axon extension occurs independently of centrosomal microtubule nucleation. Science 327:704-707.

Tanaka Y, Kanai Y, Okada Y, Nonaka S, Takeda S, Harada A, Hirokawa N (1998) Targeted disruption of mouse conventional kinesin heavy chain, kif5B, results in abnormal perinuclear clustering of mitochondria. Cell 93:1147-1158.

Thangavel R, Sahu SK, Van Hoesen GW, Zaheer A (2009) Loss of nonphosphorylated neurofilament immunoreactivity in temporal cortical areas in Alzheimer's disease. Neuroscience 160:427-433.

Tian G, Jaglin XH, Keays DA, Francis F, Chelly J, Cowan NJ (2010) Disease-associated mutations in TUBA1A result in a spectrum of defects in the tubulin folding and heterodimer assembly pathway. Hum Mol Genet 19:3599-3613.

Tintignac LA, Brenner HR, Rüegg MA (2015) Mechanisms regulating neuromuscular junction development and function and causes of muscle wasting. Physiol Rev 95:809-852.

Vallee RB (1982) A taxol-dependent procedure for the isolation of microtubules and microtubule-associated proteins (MAPs). J Cell Biol 92:435-442.

Watson DF, Glass JD, Griffin JW (1993) Redistribution of cytoskeletal proteins in mammalian axons disconnected from their cell bodies. J Neurosci 13:4354-4360.

Yan SC, Hwang S, Rustan TD, Frey WH (1985) Human brain tubulin purification: decrease in soluble tubulin with age. Neurochem Res 10:1-18. 
Yau KW, Schatzle P, Tortosa E, Pages S, Holtmaat A, Kapitein LC, Hoogenraad CC (2016) Dendrites in vitro and in vivo contain microtubules of opposite polarity and axon formation correlates with uniform plus-end-out microtubule orientation. J Neurosci 36:1071-1085.

Yogev S, Cooper R, Fetter R, Horowitz M, Shen K (2016) Microtubule organization determines axonal transport dynamics. Neuron 92:449-460.

Yoneshima H, Yamasaki S, Voelker CC, Molnár Z, Christophe E, Audinat E, Takemoto M, Nishiwaki M, Tsuji S, Fujita I,
Yamamoto N (2006) Er81 is expressed in a subpopulation of layer 5 neurons in rodent and primate neocortices. Neuroscience 137:401-412.

Zhang Y, Chen K, Sloan SA, Bennett ML, Scholze AR, O'Keeffe S, Phatnani HP, Guarnieri P, Caneda C, Ruderisch N, Deng S, Liddelow SA, Zhang C, Daneman R, Maniatis T, Barres BA, Wu JQ (2014) An RNA-sequencing transcriptome and splicing database of glia, neurons, and vascular cells of the cerebral cortex. J Neurosci 34:11929-11947. 